\title{
MIQR: A MULTILEVEL INCOMPLETE QR PRECONDITIONER FOR LARGE SPARSE LEAST-SQUARES PROBLEMS *
}

\author{
$\mathrm{NA} \mathrm{LI} \dagger \mathrm{AND}$ YOUSEF SAAD ${ }^{\dagger}$
}

\begin{abstract}
This paper describes a Multilevel Incomplete QR (MIQR) factorization for solving large sparse least-squares problems. The algorithm builds the factorization by exploiting structural orthogonality in general sparse matrices. At any given step, the algorithm finds an independent set of columns, i.e., a set of columns that have orthogonal patterns. The other columns are then block orthogonalized against columns of the independent set and the process is repeated recursively for a certain number of levels on these remaining columns. The final level matrix is processed with a standard QR or Incomplete QR factorization. Dropping strategies are employed throughout the levels in order to maintain a good level of sparsity. A few improvements to this basic scheme are explored. Among these is the relaxation of the requirement of independent sets of columns. Numerical tests are proposed which compare this scheme with the standard incomplete QR preconditioner and the robust incomplete factorization (RIF) preconditioner.
\end{abstract}

Key words. Multilevel Incomplete QR Factorization, CGLS, QR factorization, orthogonal factorization, Incomplete QR, Preconditioning, Iterative methods, Large least-squares problems, Normal equations.

AMS subject classifications. 65F10, 65F 20, 65F50.

1. Introduction. This paper considers iterative solution methods for linear least-squares problems of the form

$$
\min _{x}\|b-A x\|_{2}
$$

where $A \in \mathbb{R}^{m \times n}(m \geq n)$ is a large sparse matrix with full rank. Problems of this type arise in many scientific and engineering applications including data analysis, computational fluid dynamics, simulation, signal processing, and control problems, to name just a few. As engineers and scientists are benefitting from increased availability of data as well as computational resources, these problems are inevitably becoming harder to solve due to their size as well as their ill-conditioning. For example, the papers $[4,34]$ mention a problem of this type which arises from an animal breeding study with 60 million unknowns. In the very different area of three-dimensional computer graphics, one encounters certain least-squares problems which have complexity proportional to the number of geometry primitives [22] which, in desirable models, should include millions of polygons. Problems from such applications are usually very sparse, and can be solved iteratively or by sparse orthogonal factorizations. Iterative solution methods may have an advantage over direct methods, depending on the underlying sparsity pattern.

However, if iterative methods are to be used, then preconditioning is essential. Although it is known that iterative solution algorithms are not effective without preconditioning, there has been little effort put in developing preconditioners for least-squares problems in recent years. This is in contrast with the solution of standard (square) linear systems, where enormous progress has been made in designing both general purpose preconditioners and specialized preconditioners that are tailored to specific applications. Part of the difficulty stems from the fact that many methods solve the system (1.1) by implicitly solving the normal equations

$$
A^{T} A x=A^{T} b
$$

whose solution is the same as that of (1.1). The condition number of the coefficient matrix of normal equations system (1.2) is the square of that of the original matrix $A$. As a result, the normal equations will tend to be very ill-conditioned. In this situation preconditioning is critical for robustness. However, severe ill-conditioning of the matrix will also tend to make it difficult to obtain a good preconditioner.

Though it is possible to solve the least-squares problem (1.1) by solving normal equations (1.2), forming the system of normal equations explicitly and then solving it is not a recommended approach in general as this suffers from various numerical difficulties, see $[6,14]$ for details. For small dense problems, the best

\footnotetext{
* This work is supported by NSF grant ACI-0305120 and INT-0003274, and by the Minnesota Supercomputing Institute

${ }^{\dagger}$ Department of Computer Science and Engineering, University of Minnesota, 200 Union Street S.E., Minneapolis, MN 55455. email: $\{$ nli,saad $\} @$ cs.umn.edu
} 
overall solution method is to use a good orthogonal factorization algorithm such as the Householder QR, see, e.g., [14]. If $A=Q R$ is the "thin" QR factorization of $A$ [14], then the solution of Equation (1.1) can be obtained by solving $R x=Q^{T} b$ for x. For an comprehensive survey of direct methods, see [6].

Alternatively, iterative methods such as LSQR [27] and SOR [26] have been advocated for solving leastsquares problems of the type (1.1) when $A$ is large. A well-known approach is one that is based on solving the normal equations by the Conjugate Gradient (CG) method. The resulting algorithm is sometimes termed CGNR [31] and sometimes CGLS [6]. The latter acronym is adopted here. This paper only considers CGLS as the accelerator and focusses on developing effective preconditioners. Since we refer to preconditioned CGLS throughout the paper, we now give a brief description of the algorithm, assuming that a preconditioner $M$ for $A^{T} A$ is available. Recall that a preconditioner is a certain matrix $M$ which approximates the original coefficient matrix (in this case $A^{T} A$ ) and such that it is inexpensive to solve an arbitrary linear system $M x=b$.

Algorithm 1.1. Left-Preconditioned CGLS

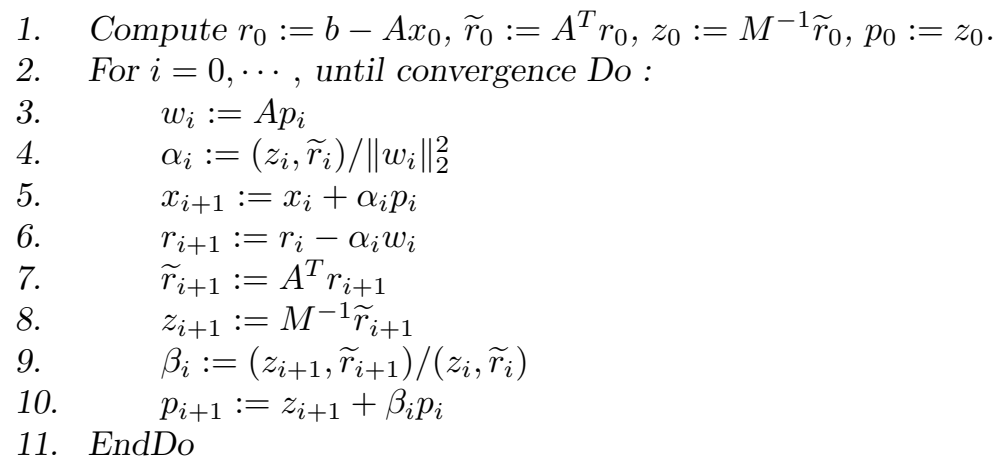

Many variants of the above algorithm exist. In particular, when $M$ is available in the form of a product $M=L L^{T}$, where $L$ is lower triangular, then the preconditioning operation can be split in two parts and a split-preconditioned CGLS option can be derived. A right-preconditioned option can be developed as well. We only consider the left-preconditioned variant in this paper.

Developing preconditioners for the normal equations, or for Problem (1.1), can be approached in a number of ways. A naive approach would be to form the squared matrix $A^{T} A$ and try to find an incomplete Cholesky factorization of this matrix. The fact that this matrix is symmetric positive definite, does not make it easy to find a preconditioner for it. Indeed, most of the theory for preconditioning techniques relies on some form of diagonal dominance. In addition, forming the normal equations suffers from other disadvantages, some of which are the same as those mentioned above for the dense case, in particular there is some loss of information when forming $A^{T} A$ [6]. Moreover, $A^{T} A$ can be much denser than the original matrix. In fact one dense row of $A$ will make the entire $A^{T} A$ matrix dense.

Another approach, one that is taken here, is to try to compute an approximate orthogonal factorization of $A$. This approach is not new as will be seen in Section 2 which discusses related work. If $A \approx Q R$, then $A^{T} A \approx R^{T} R$ and this matrix can be used as a preconditioner $M$. Notice that this approach ignores the factor $Q$ which is not used. In this paper we exploit multilevel ideas similar to those defined for the Algebraic Recursive Multilevel Solver (ARMS) in [33, 24]. The idea of Multilevel Incomplete QR (MIQR) factorization can be easily described with the help of recursion. It is important to observe at the outset that when $A$ is sparse, then many of its columns will be orthogonal because of their structure. These are called structurally orthogonal columns. It is therefore possible to find a large set $S$ of structurally orthogonal columns. This set is called an independent set of columns. Independent sets were the main ingredient used in ARMS [33, 24]. Once the first independent set $S$ is obtained, we can block orthogonalize the remaining columns against the columns in $S$. Since the matrix of the remaining columns will still be sparse in general, it is natural to think of recursively repeating the process until a small number of columns are left which can be orthogonalized with standard methods. The end result is a QR factorization of a column-permuted $A$. With this simple strategy MIQR gradually reduces a large sparse least-squares system into one with a significantly smaller size. It is worth pointing out that although we focus on overdetermined systems $(m>n)$, the techniques described are applicable to square matrices $(m=n)$ and underdetermined matrices $(m<n)$ as well.

Recent developments in the solution of standard linear systems have shown that multilevel precondi- 
tioners have excellent scalability and robustness properties, see, e.g., [33, 9, 31, 1, 2, 3]. However, it appears that when it comes to the solution of large general sparse least-squares problems, similar multilevel methods have not been considered so far, in spite of an increasing demand for solving such problems.

The remainder of this paper is organized as follows. After a short section on related work (Section 2), we discuss in Section 3 the issue of finding independent sets of columns, as this is an important ingredient used in MIQR. Then, a detailed description of MIQR is presented in Section 4 followed by strategies to improve the performance of MIQR as well as other implementation details. Numerical results are reported in Section 5 and the paper ends with concluding remarks in Section 6.

2. Related Work. Several general-purpose preconditioners based on techniques such as SSOR, incomplete orthogonal factorization, and incomplete Cholesky factorization have been proposed and analyzed in the literature.

In 1979, Björck introduced a preconditioner based on the SSOR method [5]. In the proposed method, $A^{T} A$ is written as $A^{T} A=L+D+L^{T}$, where $L$ is lower triangular. The normal equations are then preconditioned by

$$
M=\omega(2-\omega)(D+\omega L) D\left(D+\omega L^{T}\right) .
$$

To avoid forming $A^{T} A$ explicitly, row (or column) projection methods have also been exploited and applied to normal equations [10]. In these methods, only a row or a column of $A$ is needed at any given relaxation step. Block versions of these methods have also been studied $[7,19]$.

In a 1984 pioneering article, Jennings and Ajiz proposed preconditioners based on incomplete versions of Givens rotations and the Gram-Schmidt process [17]. Thereafter, several other preconditioners based on incomplete orthogonal factorizations have been studied [29, 35, 28]. If $A=Q R$ is the exact thin $\mathrm{QR}$ factorization of $A$, where $R$ is an $n \times n$ upper triangular matrix and $Q$ is an $m \times n$ orthogonal matrix, then $A^{T} A=R^{T} R$, and it is usually inexpensive to solve the equation $R^{T} R x=y$. The incomplete version of the QR factorization (IQR) can be used as a preconditioner for Equation (1.2). Unlike the matrix $Q$ produced by incomplete Givens rotations, which is always orthogonal, the factor $Q$ produced by the incomplete Gram-Schmidt process is not necessarily orthogonal. Nonetheless, the incomplete Gram-Schmidt based preconditioners are robust and can avoid breakdown when A has full rank. However, one drawback is that the incomplete Gram-Schmidt factorization requires a large intermediate storage for $Q$. In order to reduce memory requirements, dropping strategies are employed in both $Q$ and $R$. Let $P_{Q}$ and $P_{R}$ be zero patterns chosen for matrices $Q$ and $R$ respectively. The incomplete $\mathrm{QR}$ factorization based on the Gram-Schmidt process can be described by the following modification of the ILQ algorithm given in [29].

Algorithm 2.1. Incomplete QR Factorization (IQR)

1. For $j=1, \ldots, n$ Do:

2. $\quad$ Compute $r_{i j}:=\left(a_{j}, q_{i}\right)$, for $i=1,2, \cdots, j-1$,

3. Replace $r_{i j}$ by zero if $(i, j) \in P_{R}$,

4. Compute $q_{j}:=a_{j}-\sum_{i=1}^{j-1} r_{i j} q_{i}$,

5. $\quad$ Replace $q_{i j}$ by zero if $(i, j) \in P_{Q}, i=1,2, \cdots, m$

6. Compute $r_{j j}:=\left\|q_{j}\right\|_{2}$,

7. If $r_{j j}==0$ then stop; Else compute $q_{j}:=q_{j} / r_{j j}$.

8. EndDo

Similarly to dropping strategies used in ILUT [30], $P_{Q}$ and $P_{R}$ can be determined dynamically, based on the magnitude of the elements generated. In the above algorithm, the step represented by line 2 computes the inner products of the $j$-th column of $A$ with all previous columns of $Q$. Most of these inner products are equal to zero because of sparsity. Therefore, it is important to ensure that only the nonzero inner products are calculated for efficiency. The strategy proposed in [29] calculates these inner products as a linear combination of sparse vectors. Specifically, let $r_{j}=\left[r_{1 j}, r_{2 j}, \cdots, r_{j-1, j}\right]^{T}$ and $Q_{j-1}=\left[q_{1}, q_{2}, \cdots, q_{j-1}\right]$, then $r_{j}=Q_{j-1}^{T} a_{j}$ is a sparse matrix by sparse vector product. This product can be computed as a linear combination of the rows in $Q_{j-1}$, i.e., only the rows corresponding to the nonzero elements in $a_{j}$ are linearly combined. Since the matrix $Q$ is normally stored column-wise, a linked-list pointing to the elements in each row of $Q$ needs to be dynamically maintained. This strategy is also utilized in the implementation of the proposed MIQR algorithm. 
Preconditioners based on the incomplete modified Gram-Schmidt process have also been developed. The Cholesky Incomplete Modified Gram-Schmidt (CIMGS) algorithm of Wang et al. is an incomplete orthogonal factorization preconditioner based on the modified Gram-Schmidt process [35]. The paper explores rigorous strategies for defining Incomplete Cholesky factorizations, based on the relation between the Cholesky factorization of $A^{T} A$ and the QR factorization of $A$. Other authors studied direct ways to obtain the Cholesky factorization $[18,6]$. This type of approach obtains the incomplete Cholesky factorization of $C=A^{T} A$, where $C$ may or may not be formed explicitly. As an alternative, Benzi and Tůma proposed a Robust Incomplete Factorization (RIF) preconditioner which computes an incomplete $L D L^{T}$ factorization of $A^{T} A$ without explicitly forming it [4]. Their approach utilizes a conjugate Gram-Schmidt process to calculate the factorization $Z^{T} C Z=D$, where $Z$ is unit upper triangular and $D$ is diagonal. The $L D L^{T}$ factorization is obtained by letting $L^{-1}=Z^{T}$. In section 5 a few comparisons are made between this approach and the MIQR technique proposed in this paper.

There were also a number of attempts to precondition positive definite matrices which may be far from diagonally dominant. In a 1980 paper, Manteuffel [25] suggested shifting a positive definite matrix to get an incomplete Cholesky factorization. This work was pursued more recently in [32], where other diagonal shifting techniques were studied for both incomplete orthogonal factorizations and incomplete Cholesky factorizations.

The idea of utilizing independent sets of columns (rows) in the context of least-squares, or more precisely for normal equations, is not new, see, e.g., [19, 20]. The main goal of these two papers was to exploit independent sets to improve parallelism. Independent sets of columns will be the main ingredient in obtaining an MIQR factorization. In terms of parallel algorithms, Elmroth and Gustavson developed recursive parallel QR factorizations that can be used in direct solvers for dense normal equations [12, 13].

3. Independent Sets of Columns. The MIQR algorithm proposed in this paper exploits successive independent sets of columns. This section discusses column independent set orderings.

Given a matrix $A=\left[a_{1}, a_{2}, \cdots, a_{n}\right]$, where $a_{1}, a_{2}, \cdots, a_{n}$ are column vectors, a subset $\left\{a_{j_{1}}, a_{j_{2}}, \cdots, a_{j_{s}}\right\}$ is called an independent set of columns of $A$ if columns $l$ and $k$ of $A$ are structurally orthogonal for any $l, k \in\left\{j_{1}, j_{2}, \cdots, j_{s}\right\}$ and $l \neq k$. Figure 3.1(a) shows an example of such an independent set of five columns (marked as open circles). The issue of finding independent sets of columns is not new and has been discussed in depth in the literature in different contexts, see, e.g. [11, 21, 23, 31]. Here, we formalize the problem into that of finding an independent set in a graph.

3.1. Finding Independent Set of Columns. Two columns $a_{i}$ and $a_{j}$ of $A$ will be said to be adjacent if their patterns overlap. This means that if $\hat{a}_{k}$ is the column vector obtained from $a_{k}$ by replacing all its nonzero entries by ones, then $a_{i}$ and $a_{j}$ are adjacent iff $\hat{a}_{i}^{T} \hat{a}_{j} \neq 0$. The opposite of adjacent is structurally orthogonal: two columns $a_{i}$ and $a_{j}$ are structurally orthogonal if $\hat{a}_{i}^{T} \hat{a}_{j}=0$.

Let $\hat{A}$ be the pattern matrix obtained from $A$ by replacing all its nonzero entries by ones. We call Column Orthogonality Graph (COG) of $A$ the graph with $n$ vertices representing the $n$ columns of $A$, and with edges defined by the nonzero pattern of $\hat{A}^{T} \hat{A}$. This means that there is an edge between vertex $i$ and $j$ iff $\hat{a}_{i}$ and $\hat{a}_{j}$ are adjacent.

Note that an edge from vertex $i$ to vertex $j$ is defined if $\cos \theta_{i j} \neq 0$, where $\theta_{i j}$ is the angle between vectors $\hat{a}_{i}$ and $\hat{a}_{j}$. Define the following matrices,

$$
B=\left[\frac{\hat{a}_{1}}{\left\|\hat{a}_{1}\right\|}, \frac{\hat{a}_{2}}{\left\|\hat{a}_{2}\right\|}, \cdots, \frac{\hat{a}_{n}}{\left\|\hat{a}_{n}\right\|}\right] \text { and } C=B^{T} B .
$$

Since the generic entry $c_{i j}$ of $C$ can be written as $c_{i j} \equiv \cos \theta_{i j}$, then the graph $C O G(A)$ is nothing but the adjacency graph of $C$. Therefore, the problem is to find a maximal independent set of a graph. Let $E$ be the set of all edges in $C O G(A)$ and $V$ the set of its vertices. Recall that an independent set $S$ is a subset of the vertex set $V$ such that

$$
\text { if } x \in S \text {, then }(x, y) \in E \text { or }(y, x) \in E \Rightarrow y \notin S \text {, }
$$

i.e., any vertex in $S$ is not allowed to be adjacent with any other vertex in $S$ either by incoming or outgoing edges. An independent set $S$ is maximal if

$$
S^{\prime} \supseteq S \text { is an independent set } \Rightarrow S^{\prime}=S \text {. }
$$

Note that the maximal independent set is not necessarily the independent set with maximum cardinality. In fact, to find the latter is NP hard. In the following, the term independent set will always mean a 


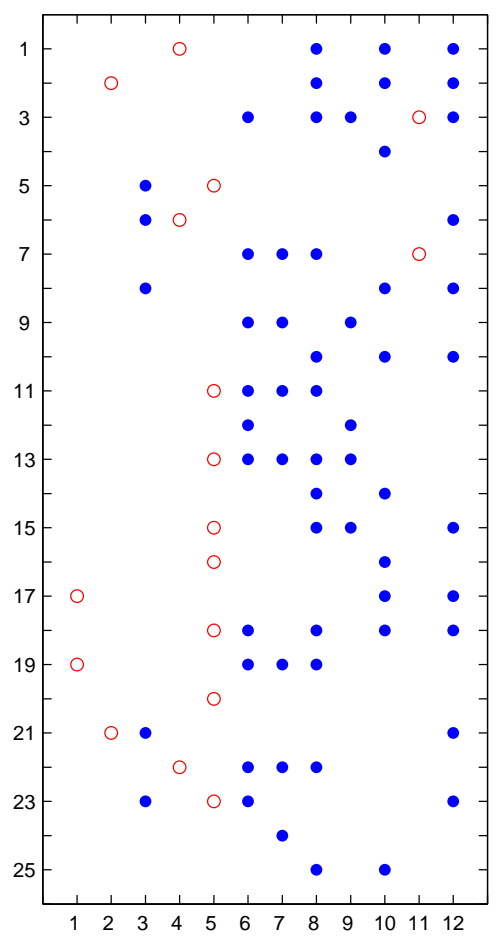

(a)

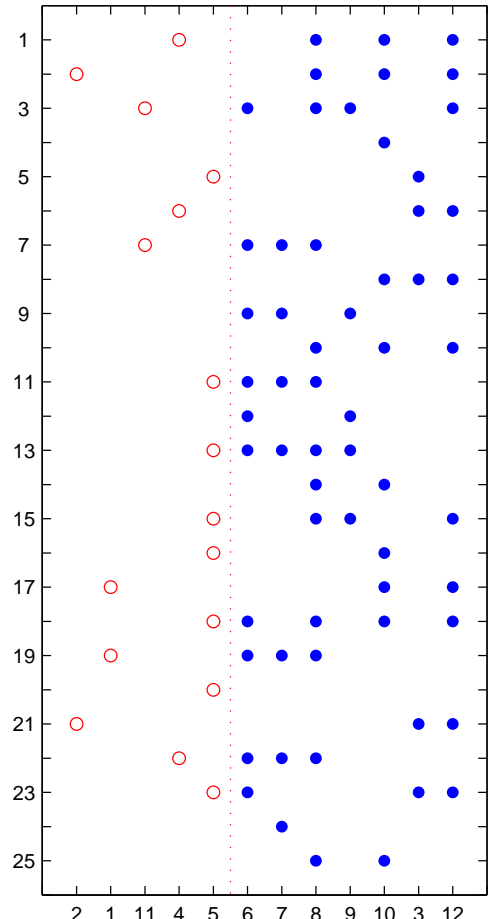

(b)

FIG. 3.1. (a) An independent set of five columns (open circles) in a $25 \times 12$ matrix. (b) The independent set of columns are permuted to the first five columns of the matrix.

maximal independent set. The following greedy algorithm, see e.g., [31], can be used to find an independent set $S$. In the algorithm, $U$ is the set of all unmarked vertices, which initially includes all the vertices.

Algorithm 3.1. Independent set ordering

1. Let $S:=\phi$ and $U:=\{1,2, \cdots, n\} . j:=1$.

2. Do While $U \neq \phi$ and $j \leq$ max Steps:

3. Let $k:=$ next unmarked vertex in $U$.

4. $S:=S \cup\{k\}$. Mark and remove $k$ from $U$.

5. Mark all vertices adjacent to $k$ and remove them from $U$.

6. $j:=j+1$.

7. EndDo

Let $|S|$ be the size of $S$. Assume that the maximum degree of all vertices in $S$ is $d_{S}$. According to Algorithm 3.1, the total number of vertices marked in line 5 is $n-|S|$. At the same time, whenever a vertex is added to $S$, at most $d_{S}$ vertices will be marked, which means the total number of vertices marked is at most $d_{S}|S|$. Therefore, we have $n-|S| \leq d_{S}|S|$ and as a result

$$
|S| \geq \frac{n}{1+d_{S}}
$$

This suggests that we may obtain $S$ with a larger number of vertices by visiting first the vertices with smaller degrees [31].

Algorithm 3.2. Independent set ordering with increasing degree traversal

1. Find an ordering $i_{1}, i_{2}, \cdots, i_{n}$ of the vertices by increasing degree.

2. Let $S:=\phi$ and $U:=\left\{i_{1}, i_{2}, \cdots, i_{n}\right\} . j:=1$.

3. While $U \neq \phi$ and $j \leq \max$ Steps:

4. Let $i_{k}:=$ next unmarked vertex in $U$. 
5. $\quad S:=S \cup\left\{i_{k}\right\}$. Mark and remove $i_{k}$ from $U$.

6. Mark all $i_{k}$ 's adjacent vertices and remove them from $U$.

7. $j:=j+1$.

8. EndDo

Algorithm 3.2 first sorts the vertices in increasing degree order, and then applies the greedy algorithm. In general, Algorithm 3.2 will find a larger size independent set at the cost of an initial sorting of the vertices. Algorithm 3.2 is used in the implementation of MIQR.

3.2. Estimates for the size of the independent set. The lower bound of the independent set size given by (3.2) is a rough one. The goal of this section is to find a more accurate estimate of the size of the independent set using a simple probabilistic model.

Consider an $m \times n$ sparse matrix $A$ with $N_{n z}$ nonzero entries, and assume that these nonzero entries are randomly distributed. In particular each column will have on average the same number of nonzero entries which is $\nu \equiv N_{n z} / n$. Under this assumption, Algorithms 3.1 and Algorithm 3.2 would be equivalent and therefore, we can restrict our study to Algorithm 3.1. We denote by $\mu$ the average number of nonzero entries per row, so $\mu \equiv N_{n z} / m$.

For any column vector $a$ of $A$, we first calculate the expected number of column vectors that are not structurally orthogonal to $a$. If $a$ has only one nonzero element, then there are on average $n-1-(\mu-1)=n-\mu$ possible columns among $n-1$ that will be orthogonal to $a$, so the probability that any given column be orthogonal to $a$ is $(n-\mu) /(n-1)$. Since $a$ has $v$ nonzero elements on average, the probability that any given column be orthogonal to $a$ is

$$
p=\left(\frac{n-\mu}{n-1}\right)^{\nu}
$$

As a result the probability that any given column is not orthogonal to $a$ is $1-p$. Thus, the expected number of column vectors that are not structurally orthogonal to $a$ is

$$
\eta=(n-1) \times\left(1-\left(\frac{n-\mu}{n-1}\right)^{\nu}\right) .
$$

Note that $\eta$ is simply the average degree of a node in $C O G(A)$ in the very first step of Algorithm 3.1, since it represents the average number of columns that are not orthogonal to a given column of $A$.

Consider now an arbitrary step $j$ of Algorithm 3.1. We will call $N_{j}$ the number of columns left to be considered, i.e., the number of unmarked columns in $U$ at the end of step $j$ of Algorithm 3.1. Initially $N_{0}=n$. The notation simplifies somewhat if we also define $n_{j} \equiv N_{j}-1$.

LEMma 3.1. Let $N_{j}$ be the expected number of unmarked columns at the end of the $j$-th step of Algorithm 3.1 and let $n_{j} \equiv N_{j}-1$. Then $n_{j}$ satisfies the following recurrence relation

$$
n_{j}=\left(\left(1-\frac{\nu}{m}\right)\left(1+\frac{1}{n_{j-1}}\right)\right)^{\nu} n_{j-1}-1
$$

Proof. We begin by observing that if we consider the matrix consisting of the unmarked columns of $A$ at any given step then its average number of nonzero entries per column remains unchanged and equal to $\nu$. In contrast, the removal of one column will change $\mu$. If $\mu_{j}$ is the average number of nonzero entries per row for the matrix of unmarked columns, then

$$
\mu_{j}=\frac{\nu \times N_{j}}{m}=\frac{\left(n_{j}+1\right) \nu}{m} .
$$

Assume that the independent set obtained is $S=\left\{i_{1}, i_{2}, \cdots, i_{s}\right\}$, where $i_{1}$ is the first vertex added into $S, i_{2}$ is the second vertex added into $S$, and so on. When $i_{j}$ is added, the estimated number of vertices that are newly marked in line 5 in Algorithm 3.1 is simply the expected number of columns that are not orthogonal to a given column for the matrix of unmarked columns. This is simply the expression (3.4) in the very first 
step, i.e., when $j=1$. For a general step it will be the same expression with $n$ replaced by $N_{j}$ and $\mu$ by $\mu_{j}$. Note that $i_{j}$ itself is also marked. The new number of unmarked columns is therefore

$$
N_{j}=N_{j-1}-1-\left(N_{j-1}-1\right) \times\left(1-\left(\frac{N_{j-1}-\mu_{j-1}}{N_{j-1}-1}\right)^{\nu}\right)
$$

This yields the following expression for $n_{j}$

$$
n_{j}=\left(\frac{n_{j-1}+1-\mu_{j-1}}{n_{j-1}}\right)^{\nu} n_{j-1}-1=\left(1-\frac{\mu_{j-1}-1}{n_{j-1}}\right)^{\nu} n_{j-1}-1
$$

Substituting, $\mu_{j-1}$ given by (3.6) yields the expression

$$
\begin{aligned}
n_{j} & =\left(1-\frac{\frac{\left(n_{j-1}+1\right) \nu}{m}-1}{n_{j-1}}\right)^{\nu} n_{j-1}-1=\left(1-\frac{\nu}{m}+\frac{1-\frac{\nu}{m}}{n_{j-1}}\right)^{\nu} n_{j-1}-1 \\
& =\left(\left(1-\frac{\nu}{m}\right)\left(1+\frac{1}{n_{j-1}}\right)\right)^{\nu} n_{j-1}-1
\end{aligned}
$$

which is the expression to be proved.

The important point about the expression (3.5) is that it is exact. However, it does not seem possible to obtain a simple closed form expression for $n_{j}$. One would be tempted to make the approximation $1 / n_{j} \approx 0$ but this is not valid since toward the end $n_{j}$ will become small. On the other hand, one can find rough bounds for $n_{j}$ and substitute them above.

Thus, since $n \geq n_{j}$ for all $j$ we have

$$
n_{j} \geq[(1-\nu / m)(1+1 / n)]^{\nu} n_{j-1}-1 .
$$

Let

$$
\alpha \equiv[(1-\nu / m)(1+1 / n)]^{\nu} \quad \text { and } \quad \gamma \equiv \frac{1}{1-\alpha}
$$

Note that since $\alpha=[(1-\nu / m)(1+1 / n)]^{\nu}$ is the reduction coefficient for the case $j=1$, then $0<\alpha<1$. Then we have $n_{j} \geq \alpha n_{j-1}-1$ and since $1=\gamma-\alpha \gamma$ this becomes $\left(n_{j}+\gamma\right) \geq \alpha\left(n_{j-1}+\gamma\right)$. So,

$$
\left(n_{s}+\gamma\right) \geq \alpha\left(n_{s-1}+\gamma\right) \geq \cdots \geq \alpha^{s}\left(n_{0}+\gamma\right) \rightarrow\left(n_{s}+\gamma\right) \geq \alpha^{s}\left(n_{0}+\gamma\right)
$$

This means that $\alpha^{s} \leq\left(n_{s}+\gamma\right) /\left(n_{0}+\gamma\right)$. Noting that $n_{0}=N_{0}-1=n-1$, and defining $K=n_{s}$ we have $\alpha^{s} \leq(K+\gamma) /(n-1+\gamma)$, which upon taking logarithms and recalling that $\alpha<1$, yields,

$$
s \geq s_{\min } \equiv \frac{\log [1+(1-\alpha) K]-\log [1+(1-\alpha)(n-1)]}{\log \alpha} .
$$

The accuracy of the estimates derived above will be tested in Section 5. It will be verified in the experiments that the lower bound (3.7) is not sharp. In fact the experiments indicate, with good consistency, that a better estimate is to uses $2 s_{\min }$ as an estimate of the actual size of $s$. On the other hand the estimate given by the direct application of the formula (3.5) can be quite accurate.

4. Multilevel Incomplete QR Factorizations (MIQR). This section presents the Multilevel Incomplete QR (MIQR) preconditioning method for solving sparse least-squares systems. It begins with a discussion of the complete version of the multilevel QR factorization (Section 4.1). Then, strategies are proposed to approximate the factorization for preconditioning purposes (Section 4.2). 
4.1. Multilevel QR Factorization (MQR). When the matrix $A$ in Equation (1.1) is sparse, it will most likely have an independent set of columns $a_{j_{1}}, a_{j_{2}}, \cdots, a_{j_{s}}$. Let $P_{1}^{T}$ be the permutation matrix which permutes $a_{j_{1}}, a_{j_{2}}, \cdots, a_{j_{s}}$ into the first $s$ columns. Then we have

$$
A P_{1}^{T}=\left[A^{(1)}, A^{(2)}\right],
$$

where $A^{(1)}=\left[a_{j_{1}}, a_{j_{2}}, \cdots, a_{j_{s}}\right]$ is an $m \times s$ matrix and $A^{(2)}$ is an $m \times(n-s)$ matrix. Figure 3.1(b) shows an example of such an ordering. Without loss of generality and for simplicity, we still use $\left[a_{1}, a_{2}, \cdots, a_{s}\right]$ and $\left[a_{s+1}, a_{s+2}, \cdots, a_{n}\right]$ to denote the columns of $A^{(1)}$ and $A^{(2)}$ respectively.

Since the columns in $A^{(1)}$ are orthogonal to each other, $\left(A^{(1)}\right)^{T} A^{(1)}$ is a diagonal matrix. Then $A^{(1)}$ can be trivially factored as $A^{(1)}=Q_{1} D_{1}$ with

$$
Q_{1}=\left[\frac{a_{1}}{\left\|a_{1}\right\|_{2}}, \frac{a_{2}}{\left\|a_{2}\right\|_{2}}, \cdots, \frac{a_{s}}{\left\|a_{s}\right\|_{2}}\right], \quad \text { and } \quad D_{1}=\operatorname{diag}\left(\left\|a_{1}\right\|_{2},\left\|a_{2}\right\|_{2}, \cdots,\left\|a_{s}\right\|_{2}\right) .
$$

Let now

$$
\begin{aligned}
& F_{1}=Q_{1}^{T} A^{(2)} \\
& A_{1}=A^{(2)}-Q_{1} F_{1} .
\end{aligned}
$$

Then Equation (4.1) can be rewritten as

$$
A P_{1}^{T}=\left[A^{(1)}, A^{(2)}\right]=\left[Q_{1}, A_{1}\right]\left[\begin{array}{cc}
D_{1} & F_{1} \\
0 & I
\end{array}\right] .
$$

This is a block version of the Gram-Schmidt process, and we have

$$
Q_{1}^{T} A_{1}=0
$$

because $Q_{1}^{T} A_{1}=Q_{1}^{T}\left(A^{(2)}-Q_{1} F_{1}\right)=Q_{1}^{T} A^{(2)}-F_{1}=0$.

In the simplest one-level method, we apply a standard QR factorization to the reduced $m \times(n-s)$ system $A_{1}$ :

$$
A_{1} \widetilde{P}_{2}^{T}=Q_{2} \widetilde{R}_{2}
$$

where $\widetilde{P}_{2}^{T}$ is an $(n-s) \times(n-s)$ permutation matrix $\left(\widetilde{P}_{2}^{T}\right.$ is the identity matrix when pivoting is not used), $Q_{2}$ is an $m \times(n-s)$ orthogonal matrix and $\widetilde{R}_{2}$ is an $(n-s) \times(n-s)$ upper triangular matrix. Equation (4.2) can then be rewritten as

$$
A=\left[Q_{1}, Q_{2}\right]\left[\begin{array}{cc}
I & 0 \\
0 & \widetilde{R}_{2}
\end{array}\right]\left[\begin{array}{cc}
I & 0 \\
0 & \widetilde{P}_{2}
\end{array}\right]\left[\begin{array}{cc}
D_{1} & F_{1} \\
0 & I
\end{array}\right] P_{1}
$$

or

$$
A=Q R_{2} P_{2} R_{1} P_{1}=Q \widehat{R}
$$

if we use the following notations,

$$
Q=\left[Q_{1}, Q_{2}\right], R_{1}=\left[\begin{array}{cc}
D_{1} & F_{1} \\
0 & I
\end{array}\right], R_{2}=\left[\begin{array}{cc}
I & 0 \\
0 & \widetilde{R}_{2}
\end{array}\right], P_{2}=\left[\begin{array}{cc}
I & 0 \\
0 & \widetilde{P}_{2}
\end{array}\right] \text {, and } \widehat{R}=R_{2} P_{2} R_{1} P_{1} .
$$

If $A$ has full rank, then $\widetilde{R}_{2}$ is nonsingular. It is easy to show that $Q$ is orthogonal because

$$
Q_{1}^{T} Q_{2}=Q_{1}^{T}\left(A_{1} \widetilde{P}_{2}^{T} \widetilde{R}_{2}^{-1}\right)=\left(Q_{1}^{T} A_{1}\right) \widetilde{P}_{2}^{T} \widetilde{R}_{2}^{-1}=0 .
$$

As is the case in similar situations related to Gram-Schmidt with pivoting, the final result is equivalent to applying the standard Gram Schmidt process to a matrix obtained from $A$ by permuting its columns. 
Indeed, starting with (4.4), we have

$$
\begin{aligned}
A P_{1}^{T} & =\left[Q_{1}, Q_{2}\right]\left[\begin{array}{cc}
I & 0 \\
0 & \widetilde{R}_{2}
\end{array}\right]\left[\begin{array}{cc}
I & 0 \\
0 & \widetilde{P}_{2}
\end{array}\right]\left[\begin{array}{cc}
D_{1} & F_{1} \\
0 & I
\end{array}\right] \\
& =\left[Q_{1}, Q_{2}\right]\left[\begin{array}{cc}
I & 0 \\
0 & \widetilde{R}_{2}
\end{array}\right]\left[\begin{array}{cc}
D_{1} & F_{1} \\
0 & \widetilde{P}_{2}
\end{array}\right] \\
& =\left[Q_{1}, Q_{2}\right]\left[\begin{array}{cc}
I & 0 \\
0 & \widetilde{R}_{2}
\end{array}\right]\left[\begin{array}{cc}
D_{1} & F_{1} \widetilde{P}_{2}^{T} \\
0 & I
\end{array}\right] P_{2}
\end{aligned}
$$

which yields the following QR factorization of a column permuted $A$ :

$$
A P_{1}^{T} P_{2}^{T}=\left[Q_{1}, Q_{2}\right]\left[\begin{array}{cc}
D_{1} & F_{1} \widetilde{P}_{2}^{T} \\
0 & \widetilde{R}_{2}
\end{array}\right] .
$$

Since $A$ is sparse, $F_{1}$ and $A_{1}$ are usually sparse as well. Sparsity can also be improved by relaxing the orthogonality and applying dropping strategies in the incomplete version as will be discussed in Section 4.2. Moreover, because $A_{1}$ is likely to be still large, the above reduction process can be applied to $A_{1}$ recursively instead of obtaining its $\mathrm{QR}$ factorization with a standard algorithm. The recursion continues until the reduced matrix is small enough or the matrix cannot be further reduced.

Let $A_{0} \equiv A$. Then, generally, the factorization at levels $i=1,2, \ldots, p$ can be recursively defined as follows:

$$
A_{i-1} \widetilde{P}_{i}^{T}=\left[A_{i-1}^{(1)}, A_{i-1}^{(2)}\right]=\left[Q_{i}, A_{i}\right]\left[\begin{array}{cc}
D_{i} & F_{i} \\
0 & I
\end{array}\right]
$$

where $A_{i-1}^{(1)}$ has $s_{i}$ columns and similarly to the one-level case $\widetilde{P}_{i}^{T}$ is the column permutation which orders the set of independent columns first. Let

$$
\begin{aligned}
D_{i} & =\operatorname{diag}\left(\left\|A_{i-1}^{(1)} e_{j}\right\|_{2}\right)_{j=1, \cdots, s_{i}} \\
Q_{i} & =A_{i-1}^{(1)} D_{i}^{-1} \\
F_{i} & =Q_{i}^{T} A_{i-1}^{(2)} \\
A_{i} & =A_{i-1}^{(2)}-Q_{i} F_{i} .
\end{aligned}
$$

We will also define as before,

$$
P_{i}=\left[\begin{array}{cc}
I & 0 \\
0 & \widetilde{P}_{i}
\end{array}\right]
$$

where the identity block completes the matrix $\widetilde{P}_{i}$ into an $n \times n$ matrix.

The Multilevel QR algorithm can be simply defined as follows.

\section{Algorithm 4.1. MQR}

0. $A_{0} \equiv A$

1. For $i=1, \ldots, p$ Do

2. Compute permutation $\widetilde{P}_{i}$ and apply it to $A_{i-1}: A_{i-1} \widetilde{P}_{i}^{T}=\left[A_{i-1}^{(1)}, A_{i-1}^{(2)}\right]$

3. Compute $Q_{i}, D_{i}, F_{i}=Q_{i}^{T} A_{i-1}^{(2)}$, and $A_{i}=A_{i-1}^{(2)}-Q_{i} F_{i}$

4. EndDo

5. $\quad A_{p} \widetilde{P}_{p+1}^{T}=Q_{p+1} \widetilde{R}_{p+1}$ (Standard QR with/without pivoting)

We can now establish a result which generalizes the relation (4.6).

LEMma 4.1. At the $i$-th step of the MQR procedure, the following relation holds,

$$
A P_{1}^{T} \cdots P_{i}^{T}=\left[Q_{1}, \cdots, Q_{i} \mid A_{i}\right]\left[\begin{array}{cc}
R_{11} & R_{12} \\
0 & I
\end{array}\right]
$$


where $R_{11}$ is a (square) upper triangular matrix, with a size equal to the column size of $\left[Q_{1}, \cdots, Q_{i}\right]$.

Proof. The proof is by induction on $i$. We begin by pointing out that $\widetilde{P}_{1} \equiv P_{1}$. Since $A_{0} \equiv A$, Equation (4.7) shows that the result is trivially true for $i=1$. We now assume that (4.12) is true for $i$ and will show that it is true for $i+1$. From (4.7) we can write

$$
A_{i}=\left[Q_{i+1}, A_{i+1}\right]\left[\begin{array}{cc}
D_{i+1} & F_{i+1} \\
0 & I
\end{array}\right] \widetilde{P}_{i+1}
$$

which, when substituted in (4.12) yields,

$$
\begin{aligned}
& {\left[Q_{1}, \cdots, Q_{i}, A_{i}\right]\left[\begin{array}{cc}
R_{11} & R_{12} \\
0 & I
\end{array}\right]=\left[Q_{1}, \cdots, Q_{i},\left[Q_{i+1}, A_{i+1}\right]\left[\begin{array}{cc}
D_{i+1} & F_{i+1} \\
0 & I
\end{array}\right] \widetilde{P}_{i+1}\right]\left[\begin{array}{cc}
R_{11} & R_{12} \\
0 & I
\end{array}\right]} \\
& =\left[Q_{1}, \cdots, Q_{i},\left[Q_{i+1}, A_{i+1}\right]\right]\left[\begin{array}{cc}
R_{11} & R_{12} \\
0 & {\left[\begin{array}{cc}
D_{i+1} & F_{i+1} \\
0 & I
\end{array}\right] \widetilde{P}_{i+1}}
\end{array}\right]
\end{aligned}
$$

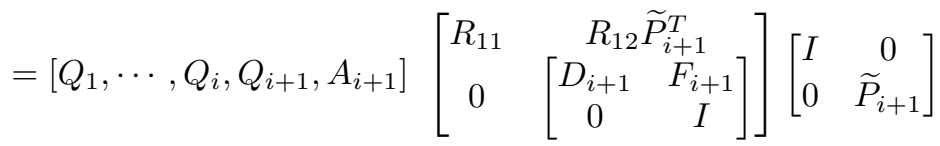

This shows that

$$
A P_{1}^{T} \cdots P_{i}^{T} P_{i+1}^{T}=\left[Q_{1}, \cdots, Q_{i}, Q_{i+1} \mid A_{i+1}\right]\left[\begin{array}{cc}
R_{11} & R_{12} \widetilde{P}_{i+1}^{T} \\
0 & {\left[\begin{array}{cc}
D_{i+1} & F_{i+1} \\
0 & I
\end{array}\right]}
\end{array}\right]
$$

which is the desired result for level $i+1$.

If the procedure stops at the $p$-th level, then $A_{p}$ is the final reduced system and we factor it as

$$
A_{p} \widetilde{P}_{p+1}^{T}=Q_{p+1} \widetilde{R}_{p+1}
$$

Note that $\widetilde{P}_{p+1}^{T}$ is the identity matrix when pivoting is not used. Then, the above lemma shows that

$$
A P_{1}^{T} \cdots P_{p}^{T} P_{p+1}^{T}=\left[Q_{1}, \cdots, Q_{p}, Q_{p+1}\right]\left[\begin{array}{cc}
R_{11} & R_{12} \widetilde{P}_{p+1}^{T} \\
0 & \widetilde{R}_{p+1}
\end{array}\right]
$$

This yields a permuted QR factorization, since it is easily shown that the columns of $\left[Q_{1}, Q_{2}, \ldots, Q_{p+1}\right]$ are orthonormal.

Lemma 4.2. Let $A$ be of full rank, and define $\mathcal{P}^{T}=P_{1}^{T} P_{2}^{T} \cdots P_{p+1}^{T}$ and $\mathcal{Q}=\left[Q_{1}, \cdots, Q_{p}, Q_{p+1}\right]$. Then $\mathcal{Q}$ is unitary and the $M Q R$ procedure computes a permuted $Q R$ factorization of $A$, i.e., we have

$$
A \mathcal{P}^{T}=\mathcal{Q R}
$$

where $\mathcal{R}$ is an upper triangular matrix.

Proof. Part of the result is established in (4.13). The only situation when the algorithm will break down is when a diagonal entry in $D_{i}$ is zero or when the last factorization fails. This is impossible in case $A$ is of full rank. It only remains to show that the matrix $\left[Q_{1}, \cdots, Q_{p}, Q_{p+1}\right]$ in (4.13) is indeed unitary. Within the same block $Q_{i}$ the columns are orthogonal structurally and they are normalized. So $Q_{i}^{T} Q_{i}=I$. For $j>i$ we have $Q_{i}^{T} Q_{j}=0$. Indeed, the columns of $Q_{j}$ are linear combinations of columns of $A_{i}$ because $j>i$. However, by construction $A_{i}$ is orthogonal to $Q_{i}$ so we have $Q_{i}^{T} Q_{j}=0$.

An illustration of the sizes and the positions of $D_{1}, F_{1}, \cdots, D_{p}, F_{p}$, and $\widetilde{R}_{p+1}$ can be visualized in Figure 4.1(a), where a four-level QR factorization process $(p=4)$ has been applied to matrix WELL1850 (Some information on this matrix can be found in Section 5). Figure 4.1(b) shows the corresponding matrix $Q=\left[Q_{1}, \cdots, Q_{p}, Q_{p+1}\right]$. Note that in order to obtain a better quality picture, the $Q$ and $R$ factors are scaled differently in the figure (the column/row size of $R$ is the same as the column size of $Q$ ). 


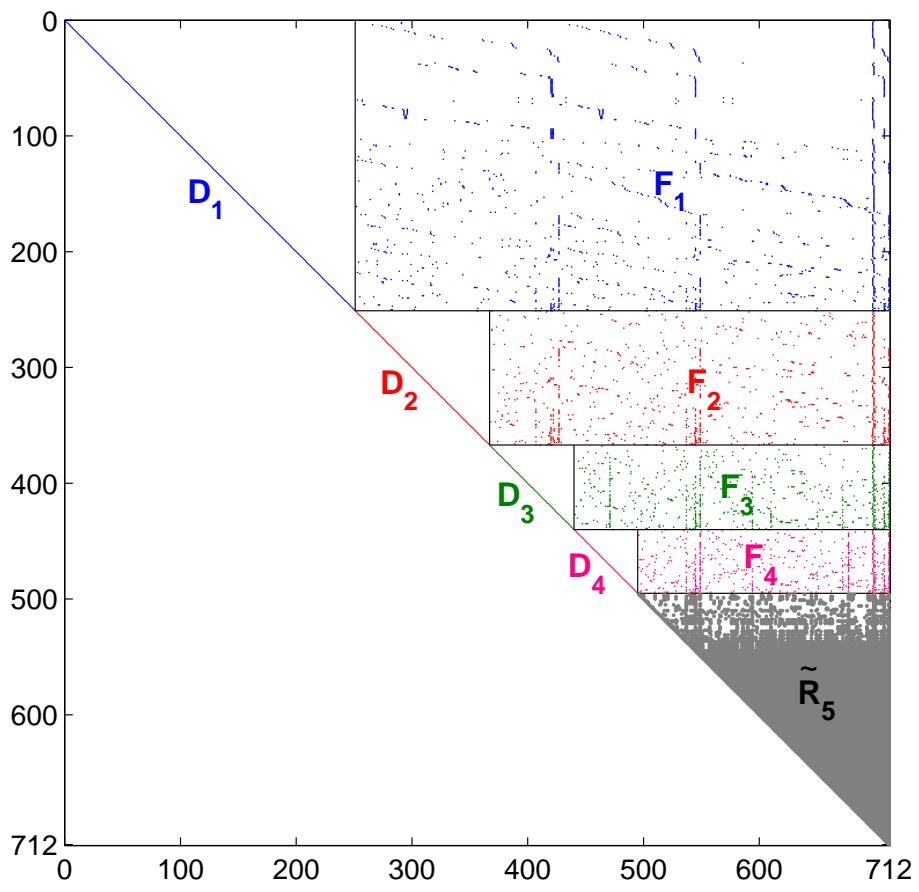

(a)

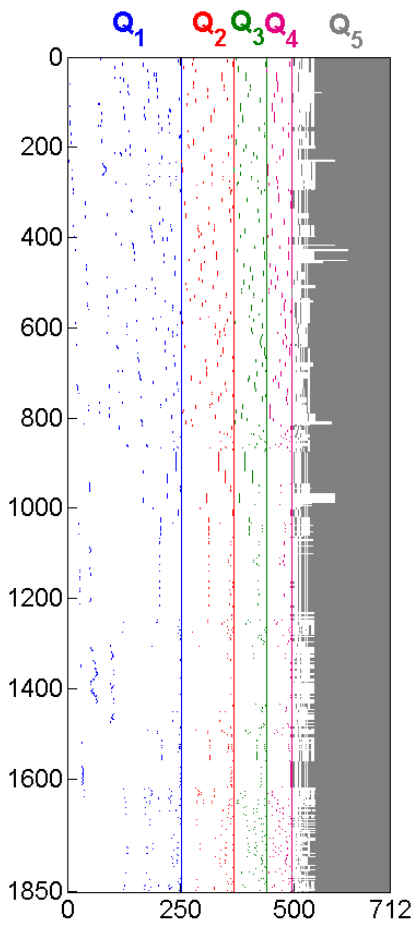

(b)

FIG. 4.1. The MQR structure for matrix WELL1850 $(1850 \times 712$, nnz $=8758)$

Another formulation of the factorization which will be used later, is to write

$$
A=Q \widehat{R}
$$

where $\widehat{R}=R_{p+1} P_{p+1} R_{p} P_{p} \cdots R_{1} P_{1} \in \mathbb{R}^{n \times n}$ and $Q=\left[Q_{1}, \cdots, Q_{p}, Q_{p+1}\right] \in \mathbb{R}^{m \times n}$. Under similar notations as the one level process, $R_{i}$ has the form

$$
R_{i}=\left[\begin{array}{ccc}
I & 0 & 0 \\
0 & D_{i} & F_{i} \\
0 & 0 & I
\end{array}\right], i=1,2, \cdots, p
$$

and

$$
R_{p+1}=\left[\begin{array}{cc}
I & 0 \\
0 & \widetilde{R}_{p+1}
\end{array}\right]
$$

4.2. Multilevel Incomplete QR Factorization. If the multilevel QR factorization is complete, we have

$$
A^{T} A=\widehat{R}^{T} \widehat{R} .
$$

Since $\widehat{R}$ is a product of permutation matrices and upper triangular matrices, it is normally inexpensive to solve the equation $\left(\widehat{R}^{T} \widehat{R}\right) x=y$. Therefore, an approximation $M \approx \widehat{R}^{T} \widehat{R}$, obtained through an incomplete multilevel QR factorization process, can be used as a preconditioner for solving Equation (1.2). In the following, a few strategies are considered for developing practical variants of the exact multilevel QR (MQR) algorithm just described. These will lead to the MIQR preconditioner.

4.2.1. Relaxed Independent Set of Columns. At each level of MQR, we would like to find a larger independent set of columns so that the reduced matrix is smaller. However, there are cases when an independent set with a large size does not even exist. For example, in an extreme case where all entries in 
one row of a matrix are nonzero, any two column vectors of the matrix are adjacent to each other, i.e., an edge exists between any two vertices in $C O G(A)$. In this case, the largest independent set will consist of one vertex only.

For the purpose of preconditioning, the orthogonality requirement can be somewhat relaxed since only an approximation of the factorization is needed. Therefore, in order to obtain a larger independent set, as well as to reduce fill-in, we will treat two column vectors as being "orthogonal" whenever the acute angle between them is "close" to a right angle. Specifically, for a given small value $\tau_{\theta}>0$, an edge from vertex $i$ to vertex $j$ is considered to belong to $C O G(A)$ if $\left|\cos \theta_{i j}\right| \geq \tau_{\theta}$. This replaces the original condition that $\cos \theta_{i j} \neq 0$. The scalar $\tau_{\theta}$ is termed the angle threshold. We denote the Column Orthogonality Graph obtained under the angle threshold $\tau_{\theta}$ by $C O G\left(A, \tau_{\theta}\right)$.

Let $C\left(\tau_{\theta}\right)$ be the matrix obtained by replacing all elements less than $\tau_{\theta}$ in absolute value in the matrix $C$ defined by (3.1) with 0. Clearly, the graph $C O G\left(A, \tau_{\theta}\right)$ is the adjacency graph of the matrix $C\left(\tau_{\theta}\right)$. Recall that the entries in $C$ (and $C\left(\tau_{\theta}\right)$ ) are cosines of columns of $B$, which represent the patterns of the columns of $A$. Alternatively, the cosines can be calculated using the real values in $A$ instead. To do so, $B=\left[\frac{a_{1}}{\left\|a_{1}\right\|}, \frac{a_{2}}{\left\|a_{2}\right\|}, \cdots, \frac{a_{n}}{\left\|a_{n}\right\|}\right]$ is used to calculate $C$ instead of $B$ given by $(3.1)$. Once $C O G\left(A, \tau_{\theta}\right)$ is obtained, Algorithm 3.1 or 3.2 can be applied to $\operatorname{COG}\left(A, \tau_{\theta}\right)$ to find an independent set. The independent set found in this way is in general significantly larger than that found by applying the same algorithm on $C O G(A)$. The effectiveness of this relaxed independent set ordering strategy is illustrated in Section 5 .

4.2.2. Dropping Strategies. The multilevel process yields denser and denser intermediate matrices $F_{i}$ in general. To ensure a moderate memory usage, we usually drop small terms from $F_{i}$. Since a relaxed orthogonality strategy is employed (see previous section), this same strategy is applied when computing the matrix $F_{i}$. Recall that $F_{i}=Q_{i}^{T} A_{i-1}^{(2)}$, where $Q_{i}$ includes normalized columns in the independent set $S$ found at level $i$, and $A_{i-1}^{(2)}$ includes all remaining columns which are not in $S$. Therefore, any element in $F_{i}$ is an inner product between a column vector in $S$ and another column vector not in $S$. For a given angle threshold $\tau_{\theta}$, the element is replaced by 0 if the cosine of the angle between these two column vectors is less than $\tau_{\theta}$ in absolute value. Assume that $F_{i}=\left\{f_{u v}\right\}, Q_{i}=\left[q_{1}, q_{2}, \cdots, q_{s}\right]$, and $A_{i-1}^{(2)}=\left[a_{1}, a_{2}, \cdots, a_{t}\right]$. Then $f_{u v}=q_{u}^{T} a_{v}=\left\|a_{v}\right\|_{2} \cos \theta_{u v}$, where $\theta_{u v}$ is the angle between $q_{u}$ and $a_{v}$. Thus, $f_{u v}$ is dropped if $\left|f_{u v}\right|<\tau_{\theta}\left\|a_{v}\right\|_{2}$.

The final reduced matrix is normally much denser than the original matrix $A$. If it is small enough (e.g., around one hundred columns), a standard QR factorization can be applied. Note that this matrix can now be treated as dense in order to take advantage of effective block computations. Otherwise, an incomplete QR factorization is applied. Although the final reduced matrix can be factorized by any incomplete QR factorization method, the incomplete Gram-Schmidt process (Algorithm 2.1) is used in our implementation. The dropping strategy used is similar to the dual threshold-based dropping strategy in ILUT. Fill-ins are dropped dynamically when the columns of $Q$ and $R$ are being formed and based on the magnitude of the columns generated.

4.2.3. MIQR. With the relaxed independent sets of columns and dropping strategies described above, the MIQR algorithm can be described as follows.

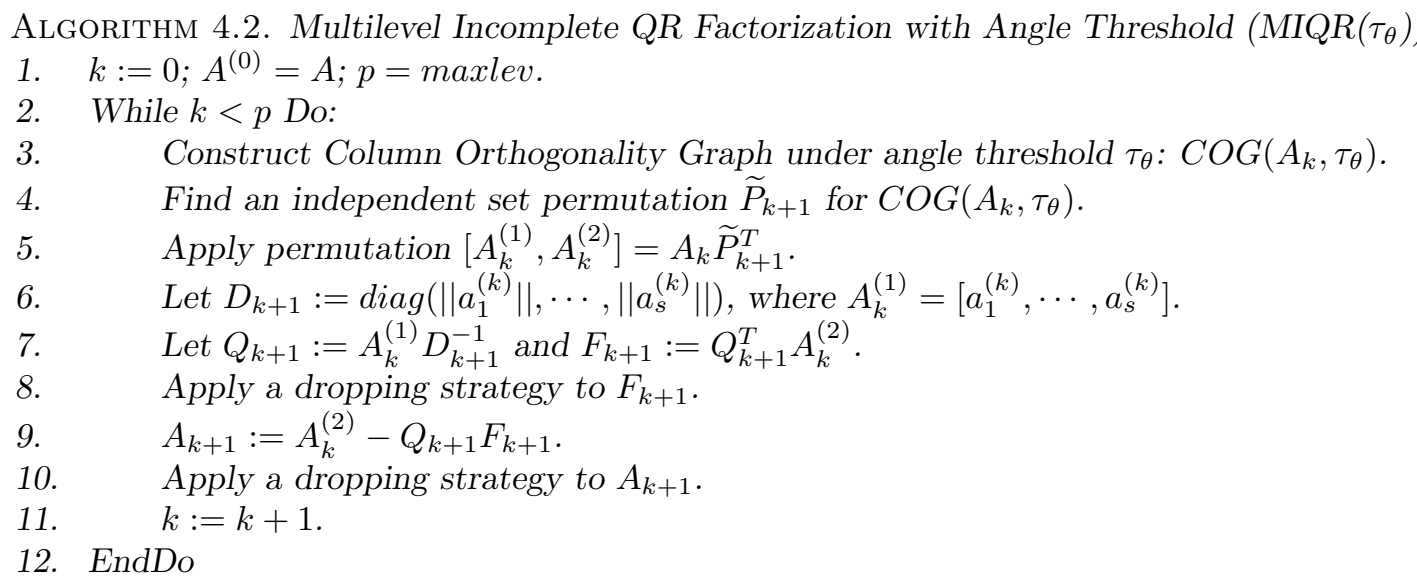


13. Apply IQR (or $Q R$ ) on $A_{p}: A_{p} \widetilde{P}_{p+1}^{T} \approx Q_{p+1} \widetilde{R}_{p+1}$

Some implementation details are now discussed. Theoretically, we can continue the multilevel incomplete QR factorization process until the reduced matrix is very small or the system cannot be further reduced. Practically, the overhead of the multilevel process increases substantially as more levels are taken. At the same time, since the multilevel process yields denser and denser matrices, the number of independent columns available becomes much smaller. Therefore, it is best to stop the multilevel process when a certain number of levels (maxlev in Algorithm 4.2) is reached or the problem size reduced is not significant (e.g. less than thirty percent of the previous problem size).

Recall that at each level $C O G\left(A, \tau_{\theta}\right)$ is the adjacency graph of $C\left(\tau_{\theta}\right)$ which is available from the matrix $C=B^{T} B$, see, (3.1). However, the matrix $C$ need not be calculated explicitly. Since we determine the degrees of the vertices one by one, only a single row of $C$ is needed at any given time. In other words, to determine the degree of vertex $i$, only the $i$-th row of $C$ needs to be calculated. This row contains the inner products between the $i$-th column of $B$ and all other columns. As indicated in [29], see also in Section 2 , these inner products can be efficiently calculated as a linear combination of the rows of $B$. For this reason, although a reduced matrix is naturally formed and stored column-wise during the multilevel QR factorization process, we maintain an index array for easily accessing its elements row-wise. Furthermore, since $C$ is symmetric, only its upper part (i.e., the inner products between the $i$-th column of $B$ and columns from $i+1$ to $n$ ) needs to be calculated.

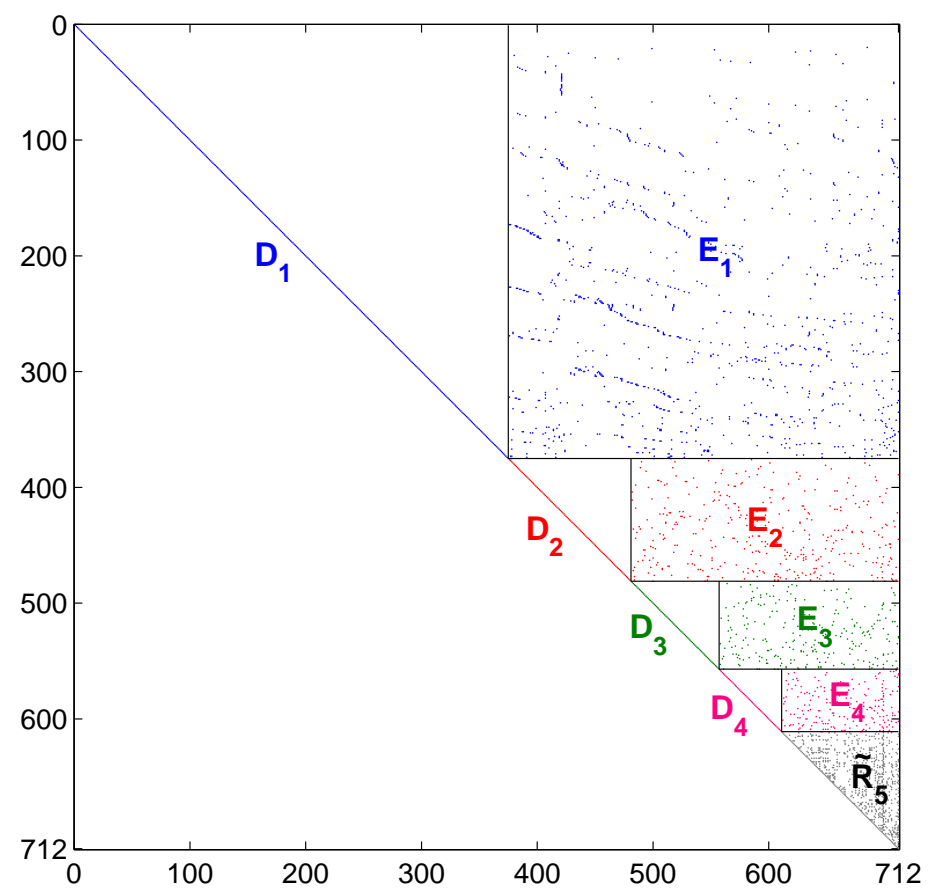

(a)

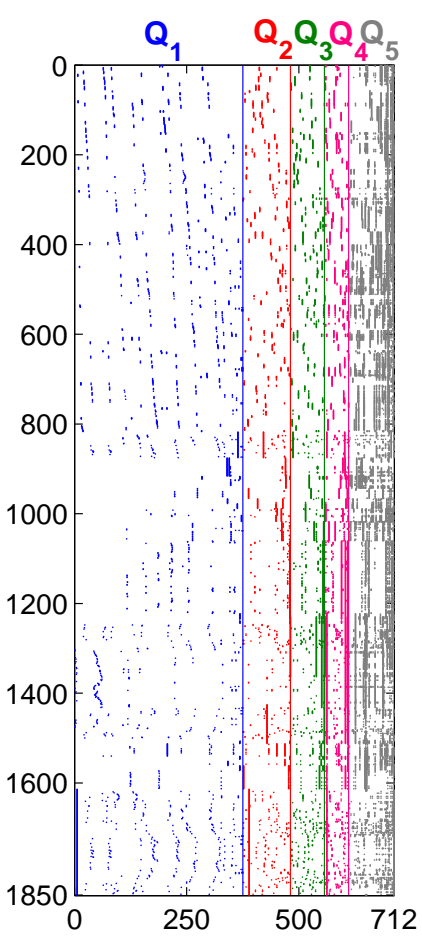

(b)

FIG. 4.2. The MIQR structure for matrix WELL1850 $(1850 \times 712$, nnz $=8758)$

As is standard practice, the permutation matrices $\widetilde{P}_{k}$ are not formed explicitly. Instead, a permutation array $\operatorname{perm}^{(k)}$ is employed to hold the new ordering of the columns at each level, along with the inverse permutation array iperm $^{(k)}$. With this strategy, the columns of matrix $A_{k}$ are kept in their original ordering and the permutation step (line 5 in Algorithm 4.2) can be avoided. To construct an MIQR preconditioner, the matrix array $\left\{D_{1}, F_{1}, D_{2}, F_{2}, \cdots, D_{p}, F_{p}, \widetilde{R}_{p+1}\right\}$ and the permutation arrays perm ${ }^{(k)}$ and iperm $^{(k)}$ are stored. Similarly to Figure 4.1, this matrix array can be organized in an $n \times n$ matrix as illustrated in Figure 4.2 , where $\tau_{\theta}=0.1$ is used and IQR is applied to the final reduced matrix. Moreover, since the matrices 
$Q_{k}$ are not needed for the preconditioning purpose, they are discarded at the end of the $k$-th level recursion respectively.

Other than the dropping strategies discussed in Section 4.2.2, an optional dropping rule may be applied to $A_{k+1}$ as well (line 10 in Algorithm 4.2). For example, any nonzero element in $A_{k+1}$ whose absolute value is less than a threshold $\tau$ times a certain norm of the column, is replaced by 0 . However, in the tests reported in Section 5, we did not apply dropping to $A_{k+1}$ (i.e., $\tau=0$ ).

As mentioned before, the matrix $\widehat{R}$ defined as in (4.15) is a product of permutation matrices and upper triangular matrices. To precondition the normal equations, we need to solve the systems $\widehat{R} x=y$ and $\widehat{R}^{T} x=y$. Let $s_{k},(k=1,2, \cdots, p)$ be the size of the independent set of columns at level $k$. Define $r_{1}=1$ and $r_{k}=r_{k-1}+s_{k-1}$ for $k=2, \cdots, p+1$. Algorithms 4.3 and 4.4 are used to solve the systems $\widehat{R} x=y$ and $\widehat{R}^{T} x=y$ respectively.
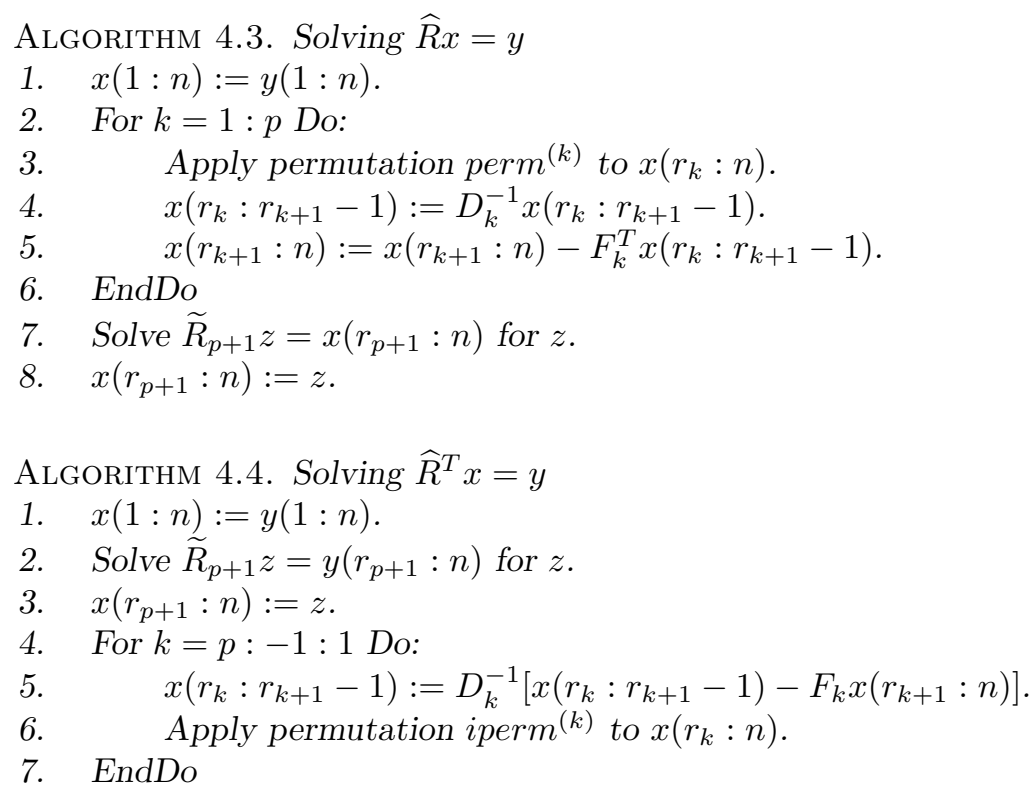

4.3. Analysis. In this section we will analyze the errors generated by the incomplete Multilevel QR factorization that are due to dropping small terms. Two questions are important to consider. The first is: how far does the result deviate from satisfying the relation $A \mathcal{P}^{T}=\mathcal{Q R}$ ? The second is: how much does $\mathcal{Q}$ deviate from orthogonality in the presence of dropping?

The basic step of MIQR can be described by approximate versions of the relations $(4.7-4.11)$. Specifically, the equations that define $F_{i}$ and $A_{i}$ will change while $Q_{i}, D_{i}$ can be assumed to be exact.

$$
\begin{aligned}
D_{i} & =\operatorname{diag}\left(\left\|A_{i-1}^{(1)} e_{j}\right\|_{2}\right)_{j=1, \cdots, s_{i}} \\
Q_{i} & =A_{i-1}^{(1)} D_{i}^{-1} \\
\widetilde{F}_{i} & =Q_{i}^{T} A_{i-1}^{(2)}+E_{F, i} \\
\widetilde{A}_{i} & =A_{i-1}^{(2)}-Q_{i} \widetilde{F}_{i}+E_{i}
\end{aligned}
$$

The term $E_{i}$ comes from dropping entries when forming the block $A_{i}$ while the term $E_{F, i}$ comes from dropping when forming the matrix $F_{i}$, see Algorithm 4.2. Then assuming $A_{i-1}$ is exact, the relation (4.7) becomes,

$$
A_{i-1} \widetilde{P}_{i}^{T}=\left[A_{i-1}^{(1)}, A_{i-1}^{(2)}\right]=\left[Q_{i}, \widetilde{A}_{i}\right]\left[\begin{array}{cc}
D_{i} & \widetilde{F}_{i} \\
0 & I
\end{array}\right]+\left[0, \quad E_{i}\right]
$$

Notice the remarkable absence of $E_{F, i}$ from the error in (4.20). The error is imbedded in $\widetilde{F}_{i}$. Before continuing, we can further notice that if there is no dropping when building $A_{i}$, then $E_{i} \equiv 0$ and the relation 
(4.7) becomes exact. This means that in the end we would expect to have the relation $A \mathcal{P}^{T}=\mathcal{Q R}$ exactly satisfied, but $\mathcal{Q}$ is not necessarily unitary. Now we consider the general case and will attempt to analyze the difference between $A \mathcal{P}^{T}$ and $\mathcal{Q R}$.

Consider the result of Lemma (4.1) which would be desirable to generalize. In the following we attempt to extend the argument used in the proof of Lemma 4.1. We write the above relation for level $i+1$ as

$$
\widetilde{A}_{i}=\left[Q_{i+1}, \widetilde{A}_{i+1}\right] \underbrace{\left[\begin{array}{cc}
D_{i+1} & \widetilde{F}_{i+1} \\
0 & I
\end{array}\right]}_{Z_{i+1}} \widetilde{P}_{i+1}+\underbrace{\left[0, E_{i+1}\right]}_{G_{i+1}} \widetilde{P}_{i+1} .
$$

Substituting in (4.12) (where $A_{i}$ is replaced by the computed $\widetilde{A}_{i}$ at the $i$-th step) yields,

$$
\begin{aligned}
& {\left[Q_{1}, \cdots, Q_{i}, \widetilde{A}_{i}\right]\left[\begin{array}{cc}
R_{11} & R_{12} \\
0 & I
\end{array}\right]=\left[Q_{1}, \cdots, Q_{i} \mid\left[Q_{i+1}, \widetilde{A}_{i+1}\right] Z_{i+1} \widetilde{P}_{i+1}+G_{i+1} \widetilde{P}_{i+1}\right]\left[\begin{array}{c|c}
R_{11} & R_{12} \\
\hline 0 & I
\end{array}\right]} \\
& =\left[Q_{1}, \cdots, Q_{i} \mid\left[Q_{i+1}, \widetilde{A}_{i+1}\right]\right]\left[\begin{array}{c|c}
R_{11} & R_{12} \\
\hline 0 & Z_{i+1} \widetilde{P}_{i+1}
\end{array}\right]+\left[\begin{array}{ll}
0, & G_{i+1} \widetilde{P}_{i+1}
\end{array}\right] \\
& =\left[Q_{1}, \cdots, Q_{i}, Q_{i+1}, \widetilde{A}_{i+1}\right]\left[\begin{array}{cc}
R_{11} & R_{12} \widetilde{P}_{i+1}^{T} \\
0 & Z_{i+1}
\end{array}\right]\left[\begin{array}{cc}
I & 0 \\
0 & \widetilde{P}_{i+1}
\end{array}\right] \\
& +\left[0, \quad G_{i+1}\right]\left[\begin{array}{cc}
I & 0 \\
0 & \widetilde{P}_{i+1}
\end{array}\right]
\end{aligned}
$$

In the sequel we will denote by $\mathcal{R}_{i}$ the matrix $\left[\begin{array}{cc}R_{11} & R_{12} \\ 0 & I\end{array}\right]$ at step $i$. So the above relation translates into

$$
\left[Q_{1}, \cdots, Q_{i}, \widetilde{A}_{i}\right] \mathcal{R}_{i}=\left[Q_{1}, \cdots, Q_{i}, Q_{i+1}, \widetilde{A}_{i+1}\right] \mathcal{R}_{i+1} P_{i+1}+\left[0, \quad G_{i+1}\right] P_{i+1}
$$

The left-hand side of the above relation is equal to $A P_{1}^{T} \cdots P_{i}^{T}+\mathcal{E}_{i}$, where $\mathcal{E}_{i}$ denotes the total error made at step $i$ of the factorization. Using a similar notation for the first term of the right-hand side will transform (4.21) into

$$
A P_{1}^{T} \cdots P_{i}^{T}+\mathcal{E}_{i}=\left(A P_{1}^{T} \cdots P_{i} P_{i+1}^{T}+\mathcal{E}_{i+1}\right) P_{i+1}+\left[\begin{array}{ll}
0 & G_{i+1}
\end{array}\right] P_{i+1}
$$

This means that $\mathcal{E}_{i}=\mathcal{E}_{i+1} P_{i+1}+\left[\begin{array}{ll}0 & G_{i+1}\end{array}\right] P_{i+1}$ and it establishes the remarkably simple recurrence relation for the total error

$$
\mathcal{E}_{i+1}=\mathcal{E}_{i} P_{i+1}^{T}-\left[0, \quad E_{i+1}\right]
$$

where the zero block in the right-hand side has the same number of columns as $\left[Q_{1}, Q_{2}, \cdots, Q_{i}\right]$. In particular we have

$$
\left\|\mathcal{E}_{p+1}\right\| \leq \sum_{i=1}^{p+1}\left\|E_{i+1}\right\|
$$

However, this inequality does not say everything about the errors. For example, it is clear that the last columns (after permutation) will undergo more perturbations than the first ones and they will therefore be less accurate. This is understandable since, for example, the columns of the first level are not perturbed by the other columns.

Consider now the accuracy of the process with respect to orthogonality. For simplicity, we will consider only the situation where there is no dropping in forming $\widetilde{A}_{i}$, i.e., the case where $E_{i}=0$. Furthermore, we also assume that the $Q_{i} \mathrm{~s}$, considered individually, are exactly orthonormal, i.e., $Q_{i}^{T} Q_{i}=I$. In this case, it is easy to see from (4.23) that the relation $A \mathcal{P}^{T}=\mathcal{Q R}$ is exactly satisfied. However, dropping in the entries of $F_{i}$ will cause loss of orthogonality. 
Consider only one step of the process. From (4.17), (4.18) and (4.19) (with $E_{i}=0$ ) we obtain,

$$
Q_{i}^{T} \widetilde{A}_{i}=Q_{i}^{T}\left(A_{i-1}^{(2)}-Q_{i} \widetilde{F}_{i}\right)=Q_{i}^{T} A_{i-1}^{(2)}-\left(Q_{i}^{T} A_{i-1}^{(2)}+E_{F, i}\right)=-E_{F, i} .
$$

Next we wish to establish a relation between this term and $Q_{i}^{T} \mathcal{Q}$. Specifically, because of the recursive nature of the algorithm, at step $i$ we have a relation similar to that given by Lemma 4.2. A little additional notation is needed. Let $\mathcal{Q}_{i}=\left[Q_{i}, \cdots, Q_{p+1}\right]$ and $\mathcal{P}_{i}^{T}=P_{i}^{T} \cdots P_{p+1}^{T}$. Then,

$$
\widetilde{A}_{i} \mathcal{P}_{i}=\mathcal{Q}_{i} \mathcal{R}_{i}
$$

where $\mathcal{R}_{i}$ is an upper triangular matrix. Multiplying to the left by $Q_{i}^{T}$ yields,

$$
Q_{i}^{T} \widetilde{A}_{i} \mathcal{P}_{i}=Q_{i}^{T} \mathcal{Q}_{i} \mathcal{R}_{i}
$$

so that

$$
-E_{F, i} \mathcal{P}_{i} \mathcal{R}_{i}^{-1}=Q_{i}^{T}\left[Q_{i}, \cdots, Q_{p+1}\right] .
$$

The above relation shows in a simple way how the error made at step $i$ will propagate to the matrices $Q_{i}^{T} Q_{j}$ for $j>i$. The result involves the inverse of an unknown triangular matrix. The matrix $\mathcal{R}_{i}$ establishes the relation between the $Q_{j}$ 's and the matrix $\widetilde{A}_{i}$. For example, for $i=1$ we would get all the matrices $Q_{1}^{T} Q_{j}$ for $j>1$ in terms of $E_{F, 1}$ the error related to dropping in the $F$ matrix in the first step.

An interesting and important question which we do not address in this paper is the issue of effective dropping. In the papers $[8,9]$, an idea was considered for dropping in such a way that the preconditioned matrix is close to the identity. This is in contrast with other methods which try to make the preconditioner close to $A$. Though this idea involves the inverse of the preconditioner, heuristics can be used to provide quite effective methods. In the context of incomplete QR, this may be doable but it will undoubtedly be more complex.

5. Numerical results. In this section, we test the performance of the MIQR method on ten leastsquares problems from real applications. Table 5.1 provides some basic information about the test matrices. In the table, $m$ is the number of rows, $n$ is the number of columns, $n n z$ is the total number of nonzeros, $\mu$ is the average number of nonzeros per row, and $\nu$ is the average number of nonzeros per column. Matrices ILLC1850 and WELL1850 are available from the Matrix Market ${ }^{1}$. The next two matrices are from a 3D mesh parameterization problem ${ }^{2}$. These matrices are generated using the method of least-squares conformal maps as described in [22]. The last six matrices (SMALL2, MEDIUM2, LARGE, LARGE2, VERYL, VERYL2) arise in animal breeding studies $[15,16]$.

We first test the accuracy of the estimate on the number of independent set of columns as described in Section 3.2. Table 5.2 shows the lower bounds (in field "New Low. $s$ ") estimated by Equation $(3.7)(K=1)$ and the values estimated by solving Equation (3.5) numerically (in field "Est. Val. $s$ ") for the ten matrices. These lower bounds and estimated values are compared with the real values calculated by Algorithm 3.2 (in field "Real Val. $s$ "). For reference, we also calculate the rough lower bounds estimated by Equation (3.2) (in field "Old Low. $s$ "), where the average degree $\eta$ (Equation (3.4)) is used as the value of $d_{S}$. It is clear that the values calculated using (3.7) provide much closer lower bounds. Recall that (3.5) and (3.7) are derived under the assumption that the nonzero elements of a matrix are randomly distributed. In spite of this assumption, the estimated values can still provide good approximations for the matrices from real applications.

Table 5.3 presents the results of finding the independent columns using different angle tolerances $\tau_{\theta}$ as described in Section 4.2.1. In the table, $\tau_{\theta}=0.00,0.05,0.10,0.15$ and 0.20 (corresponding to angles $90^{\circ}$, $87.13^{\circ}, 84.26^{\circ}, 81.37^{\circ}$, and $78.46^{\circ}$ respectively) are tested. Algorithm 3.2 is used for all tests. For each $\tau_{\theta}$, we list the number of independent columns found in the first two levels. From the table, as expected, the number of independent columns found in each level is significantly increased as the angle tolerance increases. As an

\footnotetext{
${ }^{1}$ http://math.nist.gov/MatrixMarket/

${ }^{2}$ Provided to us by Minh Nguyen from the Graphics group at the University of Minnesota, Department of Computer Science and Engineering.
} 


\begin{tabular}{|l|r|r|r|r|r|l|}
\hline Matrix & $m$ & $n$ & $n n z$ & $\mu$ & $\nu$ & Source \\
\hline ILLC1850 & 1,850 & 712 & 8,758 & 4.73 & 12.30 & Surveying \\
WELL1850 & 1,850 & 712 & 8,758 & 4.73 & 12.30 & Surveying \\
MESHPAR1 & 31,258 & 15,994 & 187,498 & 6.00 & 11.72 & 3D mesh parameterization \\
MESHPAR2 & 75,650 & 38,384 & 453,846 & 6.00 & 11.82 & 3D mesh parameterization \\
SMALL2 & 6,280 & 3,976 & 25,530 & 4.07 & 6.42 & Animal breeding \\
MEDIUM2 & 18,794 & 12,238 & 75,039 & 3.99 & 6.13 & Animal breeding \\
LARGE & 28,254 & 17,264 & 75,018 & 2.66 & 4.35 & Animal breeding \\
LARGE2 & 56,508 & 34,528 & 225,054 & 9.98 & 6.52 & Animal breeding \\
VERY & 174,193 & 105,882 & 463,303 & 2.66 & 4.38 & Animal breeding \\
VERY2 & 348,386 & 211,764 & $1,389,909$ & 3.99 & 6.56 & Animal breeding \\
\hline
\end{tabular}

TABLE 5.1

Information on the test problems: size $=m \times n ; n n z=$ the number of nonzeros; $u=$ the average number of nonzeros per row; $v=$ the average number of nonzeros per column

\begin{tabular}{|l|r|r|r|r|}
\hline Matrix & New Low. $s$ & Est. Val. $s$ & Real Val. $s$ & Old Low. $s$ \\
\hline ILLC1850 & 58 & 196 & 220 & 16 \\
WELL1850 & 58 & 196 & 237 & 16 \\
MESHPAR1 & 1,114 & 3,594 & 2,191 & 269 \\
MESHPAR2 & 2,658 & 8,613 & 5,090 & 639 \\
SMALL2 & 610 & 1,438 & 1,171 & 193 \\
MEDIUM2 & 1,974 & 4,544 & 3,297 & 633 \\
LARGE & 5,046 & 9,648 & 9,690 & 2108 \\
LARGE2 & 5,357 & 12,681 & 9,957 & 1690 \\
VERY & 30,786 & 59,020 & 60,454 & 12815 \\
VERY2 & 32,659 & 77,552 & 61,638 & 10269 \\
\hline
\end{tabular}

TABLE 5.2

Independent set sizes: comparison of the lower bounds (3.7), the estimated numbers (3.5), the actual sizes, and the rough lower bounds (3.2)

example, for matrix VERY2, without relaxing the criterion of finding independent columns (i.e., $\tau_{\theta}=0.00$ ), only 61,638 and 57,924 independent columns are found in the first two levels of reduction respectively, i.e., the problem size reduced after the first two levels is 119,562 . With the angle tolerances, the problem sizes reduced after the first two levels increase to $148,304,166,439,190,733$, and 194,724 respectively for $\tau_{\theta}=0.05$, $0.10,0.15$, and 0.20 .

Next, we test MIQR on the ten least-squares problems and compare the results with IQR (Algorithm 2.1) and RIF ([4]) preconditioners. MIQR and IQR were coded in C. RIF provided by Benzi and Tưma was in FORTRAN90. All codes were compiled in 64-bit mode with the -O2 optimization option. All experiments were performed on an IBM SP machine, which has four $222 \mathrm{MHz}$ processors sharing $16 \mathrm{~GB}$ memory. Note that we did not take advantage parallelism in all of our tests, i.e., only one of the four processors was used at a time. The right-hand sides available from the original data were used. This is in contrast from [4] where artificial right-hand sides were employed. Algorithm 1.1 with a zero initial guess was used to solve all the problems. The iterations were stopped when

$$
\left\|A^{T} b-A^{T} A x^{(k)}\right\|_{2}<10^{-8}\left\|A^{T} b-A^{T} A x^{(0)}\right\|_{2}
$$

or the maximum iteration count of 2000 was reached. To better compare the preconditioners, we use an indicator called a fill-in factor to indicate the memory usage for each method. The fill-in factor is defined as the ratio between the memory used in a preconditioner and the memory used in the original matrix. The memory used in MIQR is represented by the total number of nonzero entries in matrices $D_{1}, F_{1}, \cdots, D_{p}$, $F_{p}, \widetilde{R}_{p+1}$ as shown in Figure 4.2. We wish to compare the preconditioners under similar fill-in factors.

In Table 5.4, we test MIQR on the ten matrices under different angle tolerances $\tau_{\theta}$. In the table, "Levels" is the number of levels used, "Res.\#" is the number of columns of the final reduced matrix, "Fill- 


\begin{tabular}{|l|c|r|r|r|r|r|}
\hline Matrix & Level & $\tau_{\theta}=0.00$ & $\tau_{\theta}=0.05$ & $\tau_{\theta}=0.10$ & $\tau_{\theta}=0.15$ & $\tau_{\theta}=0.20$ \\
\hline ILLC1850 & 1 & 220 & 327 & 338 & 343 & 350 \\
& 2 & 130 & 146 & 152 & 159 & 170 \\
\hline WELL1850 & 1 & 237 & 346 & 372 & 395 & 432 \\
& 2 & 122 & 101 & 115 & 119 & 134 \\
\hline MESHPAR1 & 1 & 2,191 & 6,151 & 6,594 & 6,471 & 6,681 \\
& 2 & 2,191 & 2,483 & 3,397 & 4,213 & 5,411 \\
\hline MESHPAR2 & 1 & 5,090 & 15,685 & 16,829 & 15,818 & 15,655 \\
& 2 & 5,090 & 5,506 & 7,973 & 9,823 & 13,918 \\
\hline SMALL2 & 1 & 1,171 & 1,623 & 1,995 & 2,793 & 2,884 \\
& 2 & 1,147 & 1,022 & 1,087 & 683 & 684 \\
\hline MEDIUM2 & 1 & 3,297 & 4,836 & 6,154 & 8,092 & 8,308 \\
& 2 & 3,110 & 3,274 & 2,941 & 2,273 & 2,340 \\
\hline LARGE & 1 & 9,690 & 10,280 & 10,222 & 10,545 & 12,554 \\
& 2 & 3,794 & 4,113 & 4,563 & 4,509 & 3,320 \\
\hline LARGE2 & 1 & 9,957 & 14,681 & 18,523 & 24,994 & 25,724 \\
& 2 & 9,312 & 9,891 & 8,552 & 6,038 & 6,131 \\
\hline VERYL & 1 & 60,454 & 61,032 & 64,107 & 66,579 & 77,343 \\
& 2 & 22,095 & 23,495 & 25,781 & 25,000 & 20,203 \\
\hline VERYL2 & 1 & 61,638 & 89,500 & 114,752 & 153,992 & 157,109 \\
& 2 & 57,924 & 58,804 & 51,687 & 36,741 & 37,615 \\
\hline
\end{tabular}

TABLE 5.3

Numbers of independent columns found using different angle tolerances for the first two levels

in" is the fill-in factor, "Pre.T" is the preconditioning time in seconds, "ITS" is the number of iterations for CGLS to convergence, "Its.T" is the iteration time in seconds, and "Tot.T" is the total time in seconds. According to the table, and as expected, the size of the final reduced system decreases as the angle tolerance increases. For example, after the same number of reduction levels, the reduced system sizes for matrix MEDIUM2 are 4,724, 1,756, and 254 under angle tolerances $0.00,0.10$, and 0.25 respectively. As a result, the memory usage of MIQR decreases correspondingly. It is also noticeable that allowing more fill-ins for the MIQR preconditioner does not necessarily provide faster convergence rates. For matrix ILLC1850 as an example, it takes CGLS 290 iterations to converge under a fill-in factor of 0.668 when $\tau_{\theta}=0.00$. However, it only takes 172 iterations under a smaller fill-in factor of 0.328 when $\tau_{\theta}=0.10$. This is because the accuracy of the MIQR preconditioner is not only determined by the fill-ins allowed but by many other factors. For example, it is not known how dropping affects the orthogonality of the underlying $\mathcal{Q}$ factor. Recall that $A^{T} A-M$ is not as important as $I-M^{-1} A^{T} A$ when it comes to analyzing convergence.

We further examine the relationship between the angle tolerance, the fill-in factor, the number of iterations, and the execution times for matrix MEDIUM2 in Figure 5.1. Figure 5.1(a) shows the fill-in factors of the MIQR preconditioner as a function of the angle tolerance $\tau_{\theta}$. Figure 5.1(b) shows the number of iterations required for CGLS to converge as a function of the angle tolerance $\tau_{\theta}$. Figure 5.1(c) shows the preconditioning time, the iteration time, and the total time as a function of the angle tolerance $\tau_{\theta}$. Finally, Figure 5.1(d) shows the total time used to solve problem MEDIUM2 as a function of the fill-in factor of the MIQR preconditioner.

Table 5.5 compares MIQR with IQR and RIF. The symbol "_" in the table indicates that convergence was not obtained in 2000 iterations. Note that matrices ILLC1850, WELL1850, LARGE, and VERYL have also been tested in [4] but using artificial right-hand sides. From the table, it is clear that although the setup times of MIQR were slightly more expensive than those of IQR under similar memory usage, both the robustness and the total execution time were significantly improved. For matrices LARGE2 and VERYL2, even when much more memory was allowed, IQR failed to converge in 2000 iterations. We also observe that MIQR had better overall performances than RIF in general. For most matrices, MIQR requires fewer number of iterations to converge than that required by RIF. This is especially true for matrices MESHPAR1, MESHPAR2, and VERYL2, for which MIQR required significantly fewer iterations under similar memory 


\begin{tabular}{|l|c|r|r|r|r|r|r|r|}
\hline Matrix & $\tau_{\theta}$ & Levels & Res.\# & Fill-in & Pre.T & ITS & Its.T & Tot.T \\
\hline \multirow{3}{*}{ ILLC1850 } & 0.00 & 2 & 360 & 0.668 & 0.04 & 290 & 0.35 & 0.39 \\
& 0.10 & 4 & 77 & 0.328 & 0.04 & 172 & 0.18 & 0.22 \\
& 0.20 & 4 & 34 & 0.219 & 0.02 & 183 & 0.17 & 0.19 \\
\hline \multirow{3}{*}{ WELL1850 } & 0.00 & 2 & 353 & 0.482 & 0.04 & 85 & 0.10 & 0.14 \\
& 0.10 & 5 & 60 & 0.322 & 0.06 & 68 & 0.06 & 0.12 \\
& 0.20 & 5 & 10 & 0.224 & 0.02 & 133 & 0.12 & 0.14 \\
\hline \multirow{3}{*}{ MESHPAR1 } & 0.00 & 2 & 11,612 & 0.763 & 7.25 & 460 & 14.27 & 21.52 \\
& 0.04 & 2 & 7,536 & 0.567 & 5.52 & 405 & 10.81 & 16.33 \\
& 0.08 & 2 & 6,424 & 0.513 & 4.60 & 530 & 13.59 & 18.19 \\
\hline \multirow{3}{*}{ MESHPAR2 } & 0.00 & 3 & 25,458 & 1.097 & 34.89 & 731 & 71.13 & 106.02 \\
& 0.05 & 3 & 12,672 & 0.650 & 22.02 & 800 & 60.84 & 82.86 \\
& 0.10 & 3 & 8,938 & 0.495 & 13.00 & 1357 & 91.39 & 104.39 \\
\hline \multirow{3}{*}{ SMALL2 } & 0.00 & 3 & 1,299 & 1.073 & 0.21 & 247 & 1.19 & 1.40 \\
& 0.10 & 3 & 506 & 0.530 & 0.15 & 241 & 0.93 & 1.08 \\
& 0.20 & 3 & 132 & 0.334 & 0.08 & 284 & 0.89 & 0.97 \\
\hline \multirow{3}{*}{ MEDIUM2 } & 0.00 & 3 & 4,724 & 1.299 & 0.88 & 223 & 3.89 & 4.77 \\
& 0.10 & 3 & 1,756 & 0.628 & 0.55 & 235 & 3.03 & 3.58 \\
& 0.25 & 3 & 254 & 0.304 & 0.16 & 407 & 4.09 & 4.25 \\
\hline \multirow{2}{*}{ LARGE } & 0.00 & 3 & 2,134 & 1.247 & 0.72 & 44 & 0.86 & 1.58 \\
& 0.05 & 3 & 1,446 & 1.014 & 0.52 & 69 & 1.21 & 1.73 \\
& 0.10 & 4 & 594 & 0.867 & 2.22 & 128 & 2.12 & 4.34 \\
\hline \multirow{3}{*}{ LARGE2 } & 0.00 & 3 & 12,087 & 1.234 & 3.13 & 361 & 20.62 & 23.75 \\
& 0.10 & 3 & 3,769 & 0.515 & 2.25 & 442 & 18.29 & 20.54 \\
& 0.20 & 3 & 292 & 0.251 & 0.37 & 461 & 15.47 & 15.84 \\
\hline \multirow{2}{*}{ VERYL } & 0.00 & 4 & 8,176 & 1.120 & 6.88 & 118 & 17.11 & 23.99 \\
& 0.18 & 4 & 613 & 0.512 & 2.19 & 221 & 25.59 & 27.78 \\
& 0.20 & 4 & 259 & 0.447 & 1.56 & 196 & 21.51 & 23.07 \\
\hline \multirow{2}{*}{ VERYL2 } & 0.00 & 1 & 150,126 & 1.430 & 40.66 & 916 & 401.60 & 442.26 \\
& 0.10 & 4 & 11,586 & 0.502 & 26.50 & 497 & 157.71 & 184.21 \\
& 0.25 & 3 & 1,522 & 0.256 & 3.22 & 737 & 186.02 & 189.24 \\
\hline
\end{tabular}

TABLE 5.4

Performance of MIQR under different angle tolerances $\left(\tau_{\theta}=0.000\right.$ and $\left.\tau_{\theta}=0.015\right)$

costs.

6. Conclusion. We have presented a preconditionning technique for solving large sparse least-squares systems, which is based on a multilevel incomplete QR factorization. The algorithm exploits a divide and conquer strategy which takes advantage of structurally orthogonal columns. This allows to gradually reduce a large problem to a significantly smaller one with little computational effort. The algorithm first finds an independent set of columns, which are structurally orthogonal. The remaining columns are then orthogonalized against this first set of columns and the resulting set is orthogonalized recursively. In order to increase the size of the independent sets of columns, we proposed a strategy which consists of relaxing the orthogonality requirement. Numerical results have shown that this strategy is quite effective in finding independent column sets with large cardinality. The MIQR preconditioner has been tested and compared with a standard Incomplete QR factorization and with the Robust Incomplete Factorization (RIF). The numerical tests show that MIQR is robust and efficient. We have not implemented a parallel version of the algorithm. However, the method has been designed with parallelism in mind and a parallel implementation should scale well.

In Section 5, we have observed that the performances of MIQR may be very different when the angle tolerance varies. It remains to investigate a systematic way of selecting a good angle tolerance for a given problem. As mentioned in Section 4.2.2, the current dropping strategies at all levels use simple techniques which tend to yield a factorization that is accurate, i.e., such that $A \mathcal{P}^{T}-\mathcal{Q R}$ is small. As is the case for 


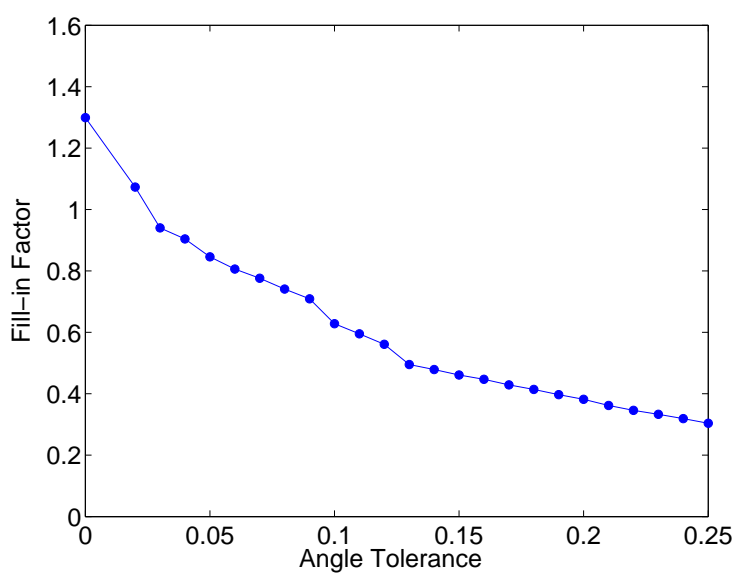

(a)

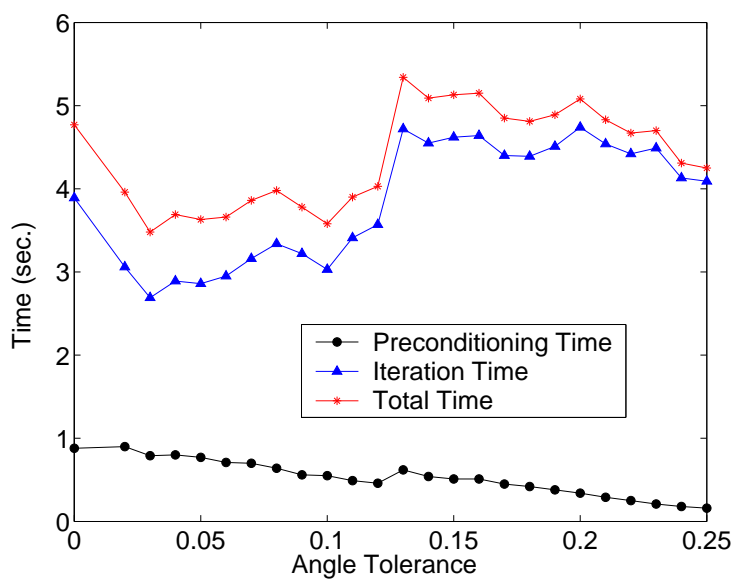

(c)

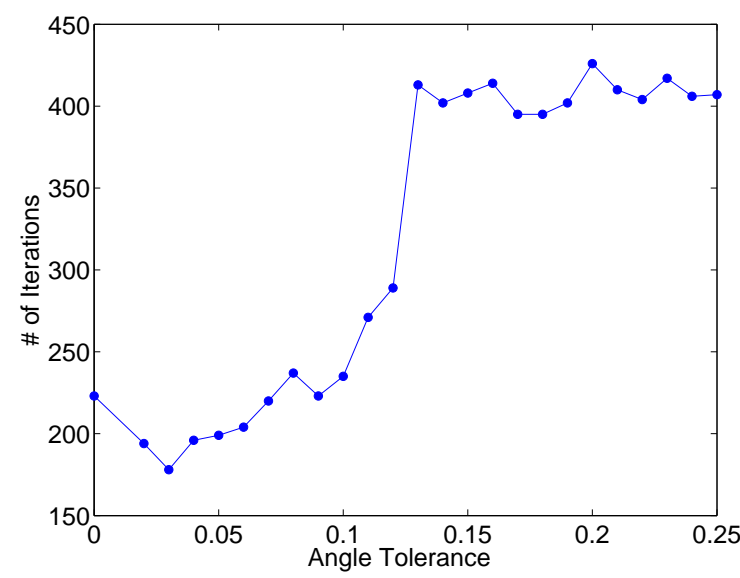

(b)

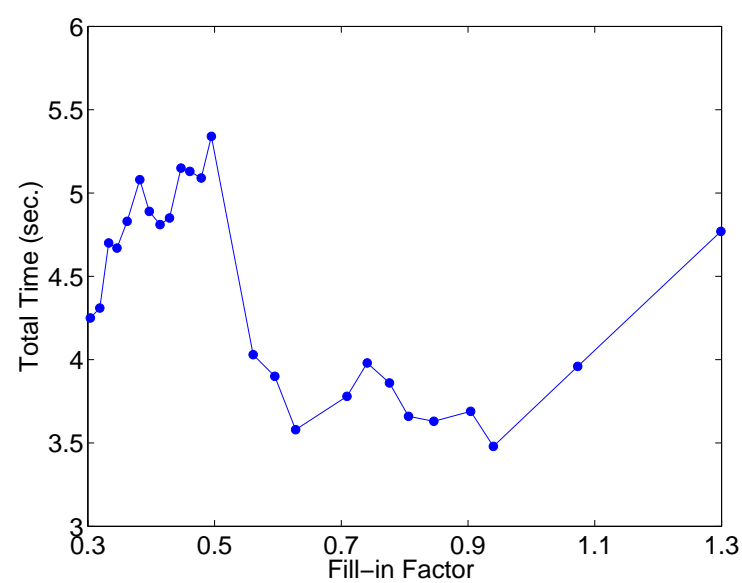

(d)

FIG. 5.1. MIQR performance on matrix MEDIUM2: (a) Fill-in factor vs. angle tolerance; (b) Total number of iterations vs. angle tolerance; (c) Preconditioning time, iteration time, and total time vs. angle tolerance; (d) Total Time vs. fill-in factor.

ILU factorizations, this is not necessarily a good strategy [8]. It may be possible to adapt Bollhöfer's work [8] to this context and develop more sophisticated dropping strategies which will in all likelihood improve the robustness of the scheme.

Acknowledgements. We would like to thank Michele Benzi and Miroslav Tůma who provided us with the RIF source code used in Section 5. We also thank Minh Nguyen for supplying us with the test matrices MESHPAR1 and MESHPAR2, and Sui Ruan for the valuable discussion on calculating the expected size of independent sets.

\section{REFERENCES}

[1] O. Axelsson and P. Vassilevski, Algebraic multilevel preconditioning methods. I., Numer. Math., 56 (1989), pp. $157-177$.

[2] —_, Algebraic multilevel preconditioning methods. II., SIAM J. Numer. Anal., 27 (1990), pp. 1569-1590.

[3] R. Bank And C. Wagner, Multilevel ILU decomposition, Numerische Mathematik, 82 (1999), pp. 543-576.

[4] M. Benzi And M. TÜma, A robust preconditioner with low memory requirements for large sparse least squares problems, SIAM J. Sci. Comput., 25 (2003), pp. 499-512.

[5] A. BJÖRCK, SSOR preconditioning methods for sparse least squares problems, in In Proceedings of the Computer Science and Statistics 12th Annual Symposium on the Interface, University of Waterloo, Canada, 1979, pp. 21-25.

[6] — Numerical Methods for Least-Squares Problems, SIAM publications, Philadelphia, PA, 1996. 


\begin{tabular}{|l|l|r|r|r|r|r|}
\hline Matrix & Method & Fill-in & Pre.T & ITS & Its.T & Tot.T \\
\hline \multirow{3}{*}{ ILLC1850 } & MIQR & 0.328 & 0.04 & 172 & 0.18 & 0.22 \\
& IQR & 0.332 & 0.02 & 1512 & 1.45 & 1.47 \\
& RIF & 0.332 & 0.19 & 406 & 0.28 & 0.47 \\
\hline \multirow{3}{*}{ WELL1850 } & MIQR & 0.322 & 0.06 & 68 & 0.06 & 0.12 \\
& IQR & 0.333 & 0.01 & 439 & 0.43 & 0.44 \\
& RIF & 0.324 & 0.21 & 90 & 0.07 & 0.28 \\
\hline \multirow{3}{*}{ MESHPAR1 } & MIQR & 0.567 & 5.52 & 405 & 10.81 & 16.33 \\
& IQR & 0.582 & 2.06 & 666 & 17.31 & 19.37 \\
& RIF & 0.586 & 3.23 & 700 & 12.44 & 15.67 \\
\hline \multirow{3}{*}{ MESHPAR2 } & MIQR & 0.650 & 22.02 & 800 & 60.84 & 82.86 \\
& IQR & 0.650 & 5.36 & 1462 & 96.27 & 101.63 \\
& RIF & 0.642 & 12.30 & 1567 & 74.14 & 86.44 \\
\hline \multirow{3}{*}{ SMALL2 } & MIQR & 0.334 & 0.08 & 284 & 0.89 & 0.97 \\
& IQR & 0.330 & 0.03 & 621 & 2.30 & 2.33 \\
& RIF & 0.321 & 6.13 & 285 & 0.75 & 6.88 \\
\hline \multirow{3}{*}{ MEDIUM2 } & MIQR & 0.304 & 0.16 & 407 & 4.09 & 4.25 \\
& IQR & 0.334 & 0.08 & 1315 & 15.93 & 16.01 \\
& RIF & 0.326 & 22.49 & 526 & 4.50 & 26.99 \\
\hline \multirow{3}{*}{ LARGE } & MIQR & 1.014 & 0.53 & 69 & 1.23 & 1.76 \\
& IQR & 1.013 & 0.43 & 157 & 3.02 & 3.45 \\
& RIF & 1.014 & 2.44 & 59 & 0.76 & 3.20 \\
\hline \multirow{3}{*}{ LARGE2 } & MIQR & 0.251 & 0.37 & 461 & 15.47 & 15.84 \\
& IQR & 0.319 & 0.22 & - & - & - \\
& RIF & 0.266 & 88.86 & 634 & 17.27 & 106.13 \\
\hline \multirow{3}{*}{ VERYL } & MIQR & 0.512 & 2.19 & 221 & 25.59 & 27.78 \\
& IQR & 0.515 & 0.70 & 997 & 117.28 & 117.98 \\
& RIF & 0.538 & 4.44 & 188 & 15.56 & 20.00 \\
\hline & MIQR & 0.256 & 3.22 & 737 & 186.02 & 189.24 \\
& IQR & 1.090 & 7.38 & - & - & - \\
& RIF & 0.288 & 1306.88 & 1220 & 226.70 & 1533.58 \\
\hline
\end{tabular}

TABLE 5.5

A comparison of $M I Q R, I Q R$, and $R I F$

[7] A. BJÖrck And T. Elfving, Accelerated projection methods for computing pseudoinverse solutions of systems of linear equations, BIT, 19 (1979), pp. 145-163.

[8] M. Bollhöfer, A robust ILU with pivoting based on monitoring the growth of the inverse factors, Linear Algebra and its Applications, 338 (2001), pp. 201-213.

[9] M. Bollhöfer AND Y. SAAD, Multilevel preconditioners constructed from inverse-based ilus, Tech. Report umsi-2004-xx2, Minnesota Supercomputer Institute, University of Minnesota, Minneapolis, MN, 2004. Submitted.

[10] R. Bramley and A. H. Sameh, Row projection methods for large nonsymmetric linear systems, SIAM J. Sci. Stat. Comput., 13 (1992), pp. 168-193.

[11] T. Coleman And J. J. Moré, Estimation of sparse Jacobian matrices and graph coloring problems, SIAM Journal on Numerical Analysis, 20 (1983), pp. 187-209.

[12] E. Elmroth And F. Gustavson, New serial and parallel recursive $Q R$ factorization algorithms for SMP systems, in PARA, 1998, pp. 120-128.

[13] — Applying recursion to serial and parallel $Q R$ factorization leads to better performance, IBM Journal of Research and Development, 44 (2000), p. 605.

[14] Gene H. Golub and Charles F. Van Loan, Matrix Computations, The Johns Hopkins University Press, Baltimore, Maryland, third ed., 1996.

[15] M. Hegland, On the computation of breeding values., in Proceedings of CONPAR 90 - VAPP IV, Joint International Conference on Vector and Parallel Processing, vol. 457, Springer, 1990, pp. 232-242.

[16] — Description and use of animal breeding data for large least squares problems, Tech. Report TR/PA/93/50, CERFACS, Toulouse, France, 1993.

[17] A. Jennings And M. A. AjIZ, Incomplete methods for solving $A^{T} A x=b$, SIAM Journal of Scientific and Statistical Computing, 5 (1984), pp. 978-987.

[18] M. Jones and P. Plassmann, An improved incomplete Cholesky factorization, ACM Transactions on Mathematical 
Software, 21 (1995), pp. 5-17.

[19] C. Kаматн, Solution of nonsymmetric systems of equations on a multiprocessor, Tech. Report 591, Center for Supercomputing Research and Development, University of Illinois at Urbana-Champaign, Aug. 1986.

[20] C. Kamath AND A. H. SAmeh, A projection method for solving nonsymmetric linear systems on multiprocessors., Parallel Computing, 9 (1989), pp. 291-312.

[21] R. LEuze, Independent set orderings for parallel matrix factorizations by Gaussian elimination, Parallel Computing, 10 (1989), pp. 177-191.

[22] B. LÉvy, S. Petitjean, N. RAy, And J. Maillot, Least squares conformal maps for automatic texture atlas generation, in Proceedings of ACM SIGGRAPH '02, ACM Press, 2002, pp. 362-371.

[23] J. G. Lewis, B. W. Peyton, and A. Pothen, A fast algorithm for reordering sparse matrices for parallel factorization, SIAM J. Sci. Stat. Comput., 10 (1989), pp. 1146-1173.

[24] Z. LI, Y. SAAD, AND M. SOSONKINA, pARMS: a parallel version of the algebraic recursive multilevel solver, Numerical Linear Algebra with Applications, 10 (2003), pp. 485-509.

[25] T. A. Manteuffel, An incomplete factorization technique for positive definite linear systems, Math. Comp., 34 (1980), pp. $473-497$.

[26] W. Niethammer, J. De Pillis, And R. VARGA, Convergence of block iterative methods applied to sparse least-squares problems, Linear Algebra Appl., 58 (1984), pp. 327-341.

[27] C. C. Paige And M. A. Saunders, Lsqr: An algorithm for sparse linear equations and sparse least squares, ACM Trans. Math. Softw., 8 (1982), pp. 43-71.

[28] A. T. Papadopoulos, I. S. Duff, And A. J. Wathen, Incomplete orthogonal factorization methods using Givens rotations. II: Implementation and results, Tech. Report 02/07, Oxford University Computing Laboratory, Mar. 2002.

[29] Y. SAAD, Preconditioning techniques for indefinite and nonsymmetric linear systems, Journal of Computational and Applied Mathematics, 24 (1988), pp. 89-105.

[30] - SPARSKIT: a basic tool kit for sparse matrix computations, Tech. Report RIACS-90-20, Research Institute for Advanced Computer Science, Moffet Field, CA, 1990.

[31] _ Iterative Methods for Sparse Linear Systems, SIAM, Philadelphia, second ed., 2003.

[32] Y. SaAd AND M. Sosonkina, Enhanced preconditioners for large sparse least squares problems, Tech. Report umsi-2001-1, Minnesota Supercomputer Institute, University of Minnesota, Minneapolis, MN, 2001. Submitted.

[33] Y. SaAd And B. Suchomel, ARMS: An algebraic recursive multilevel solver for general sparse linear systems, Numerical Linear Algebra with Applications, 9 (2002)

[34] I. Strandén, S. Tsuruta, And I. Misztal, Simple preconditioners for the conjugate gradient method: Experience with test day models, J. Anim. Breed. Genet., (2002), pp. 166-174.

[35] X. Wang, K. A. Gallivan, and R. Bramley, CIMGS: an incomplete orthogonal factorization preconditioner, SIAM J. Sci. Comput., 18 (1997), pp. 516-536. 